\title{
Inferência Bayesiana para Funções de Perdas Assimétricas
}

\author{
Gaby Rosa Amaya Robles \\ Orientador: Dr. Josemar Rodrigues
}

Dissertação apresentada ao Instituto de Ciências Matemáticas de São Carlos - USP, como parte dos requisitos para obtenção do titulo de Mestre em Ciências - Área: Ciências de Computação e Matemática Computacional.

$$
\text { USP - São Carlos }
$$

1994 
"Nunca deixe de lutar por algo que te faça ser gente, pois é a força de vontade que fará você vencer" 
En memoria a mi papá

Julio Humberto

com amor e gratitud.

Para mi mamá Arminda e hermanos

Julio y Mariano por el incentivo, compresión e amor que siempre me ofrecieron. 


\section{Agradecimentos}

A Deus por mais esta realização em minha vida.

Ao Prof. Dr. Josemar Rodrigues, pela compreensão, estimulo e sua disposição constante na orientação do presente trabalho.

Ao Prof. Dr. Jorge A. Achcar, pelo apoio desde meu ingresso no curso de pós-graduação e pelas valiosas sugestões no exame de qualificação.

À prof. Dra. Maria Creusa Breta Salles, pela amizade e apoio no ingresso ao curso de pósgraduação.

À Ana Esther Vega Vílchez, Vitoria G.Yukihara e Rumy Yamaguishi, pelo apoio e amizade.

A meus colegas, Vera, Elisa, Margareth, Claudia, Carlos Aparecido, Carlos Fogo, Marta, Silvia, Adriana, Masé, Josmar, pelo harmonioso convivio, tornando mais felizes o decorrer destes anos.

Aos professores e funcionários do ICMSC -USP.

À CAPES pelo apoio financeiro.

$\grave{A}$ todas as pessoas que direta ou indiretamente, contribuiram na realização deste trabalho. 


\section{Resumo}

Nesta dissertação, abordamos métodos Bayesianos com funções de perdas assimétricas e simétricas para comparar inferências pontuais em confiabilidade. Os resultados obtidos neste trabalho mostraram que as funções de perdas assimétricas, embora não sejam explícitas em suas soluções, são mais realísticas que as funções de perdas simétricas.

A análise Bayesiana para confiabilidade foi feita usando um modelo exponencial e algumas densidades a priori, em particular, a priori não-informativa. Também, desenvolvemos uma Função de Perda Balanceada Ponderada com censura, a qual combina a estatística clássica com a Bayesiana. Os resultados obtidos para a Função de Perda Balanceada Ponderada podem ser de grande interesse prático e metodológico 


\section{Conteúdo}

Introdução 1

1 Funções de Perdas na Teoria de Decisões 3

1.1 Introdução .............................................................................. 3

1.2 Teoria Clássica das Decisões .................................................................. 3

1.3 Elementos de um Problema de Decisão Estatística....................................... 4

1.4 Conceitos Básicos da Teoria de Decisão Estatística .................................. 5

1.5 Funções de Perdas na Teoria Clássica de Decisões....................................... 6

1.5.1 Função de Perda Quadrática ........................................................ 6

1.5.2 Função de Perda Linear .......................................................... 8

1.5.3 Função de Perda "0 - 1"................................................................. 8

1.6 Teoria da Decisão Bayesiana ............................................................. 9

1.7 Formulação do Teorema de Bayes.......................................................... 10

1.8 Elementos Básicos na Teoria de Decisão Bayesiana ..................................... 16

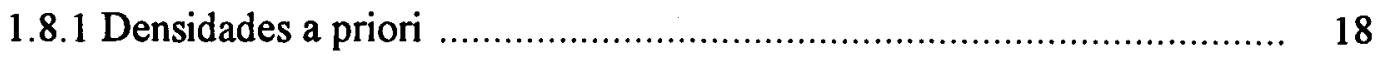

1.9 Funções de Perdas na Teoria de Decisão Bayesiana ....................................... 19

2 Estimação do Parâmetro de uma Distribuição Exponencial 24

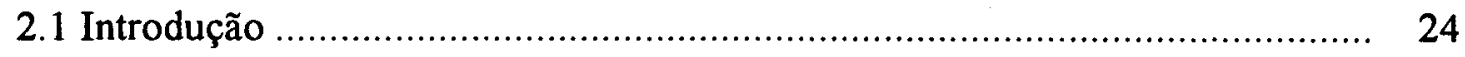

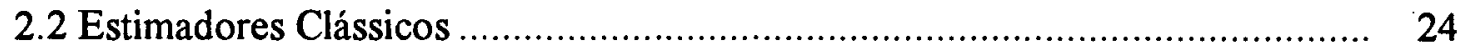

2.3 Estimadores Bayesianos ................................................................ 31

2.4 Comparação entre o Método Clássico e Bayesiano ..................................... 35 


\begin{abstract}
In this dissertation, we approach Bayesian methods with symmetrical or asymmetrical loss functions for comparison of pontual inferences in reliability. The results obtained here show that asymmetrical loss functions are more realistic than symmetrical loss functions.

Bayesian reliability analysis using the exponencial model is considered and some prior density functions have been used, in particulary, non-informative priors. Also, it was developed a weighted balanced loss functions with censoring which combine classical and Bayesian statistics. The results obtained for weighted balanced loss functions may be of great practical and methodological interest.
\end{abstract}


3 Estimação Bayesiana na Confiabilidade Usando uma Função de Perda Assimétrica

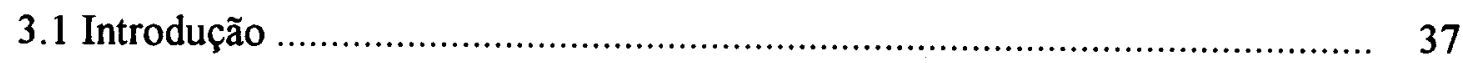

3.2 Formulação do Modelo Exponencial.......................................................... 37

3.3 Função de Perda Assimétrica ........................................................................ 39

3.4 Densidade a Priori ............................................................................... 41

3.5 Densidade a Posteriori.................................................................................. 42

3.6 Estimação da Confiabilidade ....................................................................... 42

4 Estimação Bayesiana Utilizando uma Função de Perda Linex 48

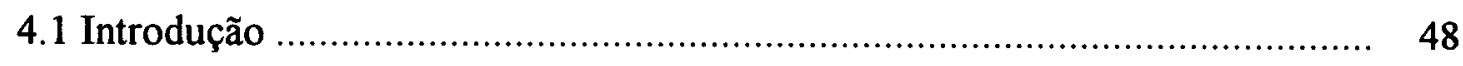

4.2 Função de Perda Linex .......................................................................... 49

4.3 O Estimador de Bayes para $\theta$................................................................. $\quad 50$

4.4 O Estimador de Bayes para $\theta$ Utilizando uma Densidade a Priori

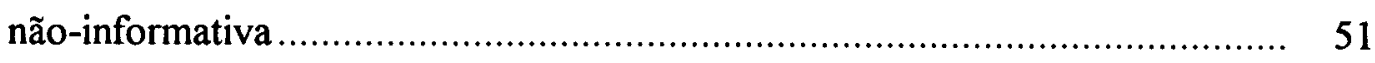

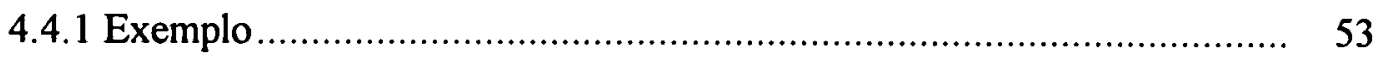

4.5 O Estimador de Bayes para $\theta$ Utilizando uma Densidade a Priori Gama

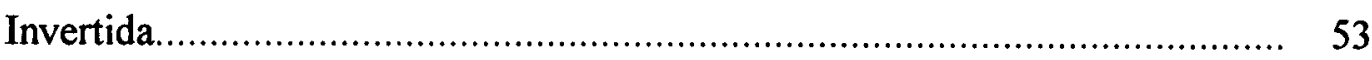

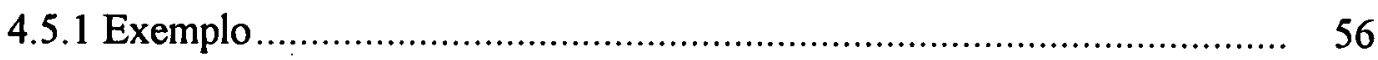

4.6 O Estimador de Bayes para $\theta$ Utilizando uma Densidade a Priori Uniforme.... 57

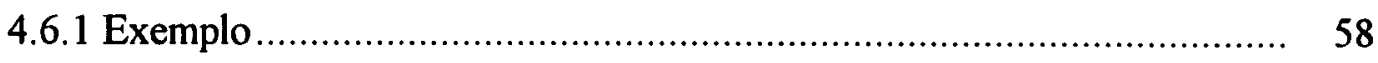

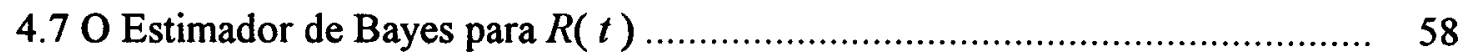

4.7.1 O Estimador de Bayes Utilizando uma Densidade a Priori

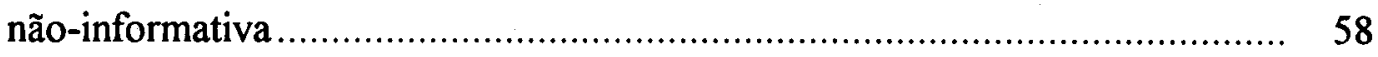

4.7.2 O Estimador de Bayes Utilizando uma Densidade a Priori Beta $(\alpha, \beta)$.. 61

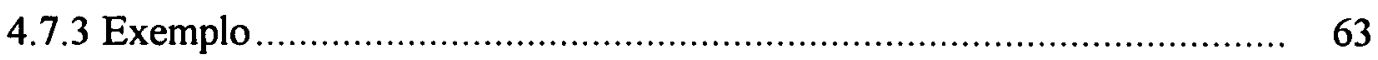

5 Função de Perda Balanceada Ponderada com Dados Censurados 64

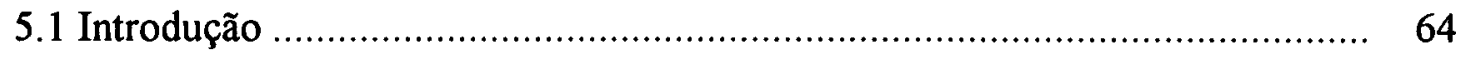


5.2 Obtenção do Estimador Ótimo Utilizando a Função de Perda Balanceada Ponderada (FPBP) 65

5.3 Comparação do Estimador Ótimo Balanceado com o Estimador Clássico 68

5.4 Conclusão 70

6 Conclusões e Perspectivas Futuras Apêndices

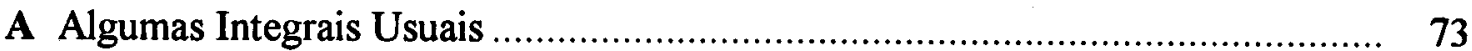

B Algoritmo Newton-Rapshon ............................................................ 74

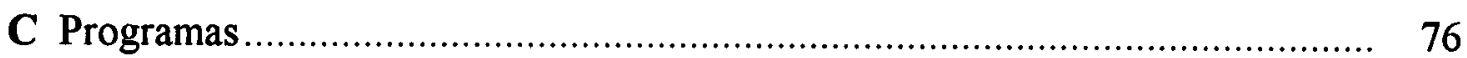

$\begin{array}{lc}\text { Referências Bibliográficas } & \mathbf{8 0}\end{array}$ 


\section{Introdução}

A teoria das decisões é uma área da estatística clássica interessada na formulação de procedimentos de decisão onde o componente estocástico desempenha um papel crucial. A teoria clássica das decisões (baseada na freqüência relativa de experimentos similares) foi originalmente desenvolvida por Abraham Wald (1950). Um elemento básico dessa teoria é a função de perda, que permite medir o quanto perdemos ao tomar uma determinada decisão sem nos preocuparnos com o ajuste do modelo adotado.

Um método que atualmente está sendo muito utilizado na solução de problemas reais é o método Bayesiano, o qual consiste em tomar decisões baseadas em dados passados (priori) e dados presentes (amostra). Os métodos Bayesianos tem sido propostos e desenvolvidos para uma ampla variedade de problemas de confiabilidade, principalmente na área industrial. Um destes problemas é procurar funções de perdas que comparada com a usual perda quadrática nos proporcione soluções mais realísticas em termos de eficiência relativa e custos.

Em geral, os procedimentos de estimação existentes na literatura estatística estão baseados na função de perda quadrática ou função de perda simétrica. Entretanto, segundo alguns autores, como veremos no desenvolvimento deste trabalho, as funções de perdas simétricas não são adequadas para a soluções de problemas práticos. Neste caso, as funções de perdas assimétricas passam a ser mais atraentes, porém são dificeis de serem trabalhadas matematicamente.

Além das funções de perdas assimétricas, introduziremos uma função de perda denominada Função de Perda Balanceada Ponderada (FPBP), definida por RodriguesZellner (1992), a qual estabelece uma relação entre a estatística clássica e Bayesiana, simplificando de uma forma objetiva os resultados. 
Neste trabalho, apresentamos um procedimento Bayesiano baseado em funções de perdas assimétricas e Balanceada para estimar a função de confiabilidade usando especificamente um modelo exponencial.

No capítulo 1, apresentamos idéias básicas e definições da teoria clássica de decisões e Bayesiana, ilustrando esta teoria com alguns exemplos. Faremos ainda uma breve introdução sobre as funções de perdas mais conhecidas e suas principais propriedades.

No capítulo 2, utilizando alguns Lemas, obteremos, do ponto de vista clássico e Bayesiano, alguns estimadores para o parâmetro exponencial usando diferentes funções de perdas. Também apresentaremos um exemplo e concluiremos com uma breve comparação entre o método clássico e Bayesiano.

No capítulo 3, obteremos uma estimativa Bayesiana para a confiabilidade do modelo exponencial usando uma função de perda assimétrica e uma densidade a priori Beta.

No capítulo 4, serão obtidos estimadores através da função de perda Linex e a função de perda quadrática. Também, obteremos as funções de risco e risco de Bayes e faremos uma comparação entre o estimador ótimo baseado na função de perda Linex e os estimadores usuais.

No capítulo 5, obteremos o estimador ótimo de Bayes usando a Função de Perda Balanceada Ponderada e apresentaremos alguns resultados da ponderação entre o estimador clássico e Bayesiano e finalizando no capítulo 6 apresentamos conclusões e perspectivas futuras. 


\section{CAPÍTULO 1}

\section{FUNÇÕES DE PERDAS NA TEORIA DE DECISÕES}

\subsection{Introdução}

As funções de perdas são ideais nos problemas de estimação, quando se faz uso de informação amostral. A teoria clássica das decisões apresenta um estudo mais detalhado destas funções. Por outro lado, existe uma teoria alternativa que é a teoria da decisão Bayesiana, a qual utiliza informação a priori (conhecimento que tem o estatístico de experiências passadas). Neste capítulo estudaremos essas duas teorias, assim como algumas funções de perdas mais comuns na literatura.

\subsection{Teoria Clássica das Decisões}

A teoria de decisão estatística está interessada na toma de decisões baseadas no conhecimento estatístico, a qual nos permite esclarecer as incertezas (grau de conhecimento sobre os estados da natureza), envolvidas nos problemas de decisão. A maioria das incertezas podem ser consideradas como quantidades numéricas desconhecidas e são representadas pelo parâmetro $\theta$. Para fazer inferências sobre $\theta$, a estatística clássica utiliza informação amostral. Por outro lado, a teoria de decisão usa informação amostral e outros aspectos importantes do problema, a fim de tomar a melhor decisão.

A teoria clássica das decisões utiliza duas fontes de informação tipicamente importantes. A primeira fonte é o conhecimento das possíveis consequências da decisão quando se usa informação amostral. Freqüentemente, este conhecimento pode ser quantificado para determinar a perda que pode ser incorrida para cada possível decisão e para vários valores de $\theta$. Como segunda fonte, é utilizada uma informação não amostral, sendo útil porque é considerada uma informação a priori. Geralmente, a informação a priori aproxima-se de experiências passadas sobre situações similares envolvendo $\theta$. 
A incorporação de uma função de perda dentro da análise estatística foi primeiramente estudada por Abraham Wald $(1939,1950)$, sendo o mesmo considerado o fundador da teoria das decisões estatística.

Neste capítulo, serão desenvolvidos alguns conceitos mais importantes e que serão usados na teoria das decisões e que podem ser encontradas em Fergunson (1967), Lehmann (1951), Berger (1985), Brow (1984), Pericchi (1986) e Walley (1987).

\subsection{Elementos de um Problema de Decisão Estatística}

A teoria das decisões é caracterizada pelo seguintes elementos básicos:

1. O conjunto de ações : $A=\left\{a_{i}\right\}, i=1,2, \ldots$.

2. O conjunto de estados possiveis da natureza ou espaço de parâmetros: $\Theta=\left\{\theta_{j}\right\}, j=1,2, \ldots$

3. Familia de experimentos para adquirir informação (amostra) sobre $\Theta: E=\{e\}$.

4. Espaço amostral ou espaço das possíveis observações experimentais: $X=\{x\}$.

A seguir apresentaremos um exemplo para ilustrar os elementos da teoria da decisão estatística

Exemplo (Controle de Qualidade)

Uma companhia recebe um lote de 10000 transistores e contrata um estatístico, o qual designa os seguintes elementos básicos:

(a) Um espaço de ações: $A=\left\{a_{1}, a_{2}\right\}$

$a_{1}$ : Aceitar o lote e pagar o preço fixado.

$a_{2}$ : Rejeitar o lote e pagar o frete de volta.

(b) $O$ espaço de parâmetros: $\Theta=\left\{\theta_{0}, \theta_{1}, \ldots, \theta_{10000}\right\}$, onde, $\theta_{j}=\{j$ : transistores defeituosos $\}$.

(c) A familia de experimentos $E=\left\{e_{0}, e_{1}, \ldots, e_{10000}\right\}$, onde, $e_{n}=\{n$ transistores são inspecionados $\}$. 
(d) O espaço amostral para o experimento $e_{n}$ é dado por $X=\{(j, n), 0 \leq j \leq n\}$, para todo $0 \leq n \leq 10000$, onde o par $(j, n)$ é a observação de $j$ transistores defeituosos dos $n$ inspecionados .

5. A função de perda $L(\theta, a)$ : representa a perda ocorrida quando a ação $a$ é tomada e o estado da natureza é $\theta$.

6. Designação de probabilidades: uma vez decidido o experimento a variável aleatória (v.a) $x$, dado $\theta$, tem uma distribuição de probabilidades $p(x \mid \theta)$, onde,

$$
\left\{\begin{array}{l}
\sum_{i=1}^{n} p_{r}\left(x_{i} \mid \theta\right)=1 \text { se } x \text { é v.a.discreta } \\
\int_{x} p(x \mid \theta) d x=1 \text { se } x \text { é v.a. contínua. }
\end{array}\right.
$$

\subsection{Conceitos Básicos da Teoria Clássica das Decisões}

Definição 1.4.1 A esperança matemática da função $f(x, \theta)$, onde x é uma v.a., é definida por:

$$
E_{x \mid \theta} f(x, \theta)= \begin{cases}\sum_{x \in X} f(x, \theta) p(x \mid \theta) & \text { se } x \text { é v.a. discreta } \\ \int_{x} f(x, \theta) p(x \mid \theta) d x & \text { sex é v.a. contínua. }\end{cases}
$$

Definição 1.4.2 Qualquer função $\delta(x): X \rightarrow A$ é chamada regra de decisão. (aleatória ou deterministica).

Definição 1.4.3 Para uma função $\delta(x)$ a função de perda é definida por $L(\theta, \delta(x))$, onde $L(\theta, a): \theta \times a \rightarrow R, \quad a=\delta(x)$.

$L(\theta, \delta(x))$, representa a perda do estatístico ao tomar a decisão $\delta(x)$, quando o verdadeiro estado da natureza é $\theta$.

Definição 1.4.4 O risco $R(\theta, \delta)$ está definido como o valor esperado da função de perda, isto é:

$$
R(\theta, \delta)=E_{\theta}[L(\theta, \delta(x))]=\int_{x} L(\theta, \delta(x)) p(x \mid \theta) d x
$$


0 risco $R(\theta, \delta)$ é interpretado como a perda média em $x$ quando o estatístico usa a regra de decisão $\delta(x)$ e o estado da natureza $\theta$. O objetivo do estatístico é procurar um $\delta_{0}$ tal que o risco seja mínimo.

\subsection{Funções de Perdas na Teoria Clássica das Decisões}

A função de perda, tem sido utilizada também como uma função da utilidade negativa definida como:

$$
L(\theta, a)=-U(\theta, a),
$$

onde $\theta$ é o estado da natureza e $a$ ação. A função de utilidade é muito utilizada pelos economistas que estão interessados em maximizar suas utilidades para a obtenção de lucros. Por outro lado a função de perda se encarrega de minimizar as perdas em relação a seus custos.

A seguir apresentaremos os tipos de funções de perdas mais conhecidas na literatura.

\subsubsection{Função de Perda Quadrática}

A função de perda quadrática é definida como:

$$
L(\theta, a)=(\theta-a)^{2} .
$$

Há um número de motivos, porque esta perda é freqüentemente utilizada em avaliar regras de decisão. Foi originalmente usada em problemas de estimação quando os estimadores de $\theta$ são considerados não viciados. $O$ risco da perda quadrática pode ser escrito como:

$$
R(\theta, \delta)=E_{\theta}[L(\theta, \delta(x))]=E_{\theta}[\theta-\delta(x)]^{2} .
$$

Desenvolvendo o quadrado em (1.4), obtemos: 


$$
R(\theta, \delta)=\left\{\operatorname{vicio}^{2}(\theta, \delta(x))+\operatorname{var}(\delta(x))\right\}
$$

onde vício $(\theta, \delta(x))=\left(\theta-E_{\theta}[\delta(x)]\right)$. Um bom estimador, $\delta(x)$ para $\theta$, para a perda quadrática é aquele que minimiza o vício ao quadrado e sua variância, simultaneamente.

Existe uma segunda razão da popularidade da função da perda quadrática, é devido ao seu relacionamento com a teoria clássica de mínimos quadrados, que a torna tratável porque nos permite deduzir outras funções de perdas. Finalmente, para a maioria das análises de decisão, o uso da perda quadrática faz com que os cálculos sejam relativamente simples. A convexidade da perda quadrática é particularmente perturbada (grandes erros são penalizados).

Existem numerosas situações na qual a perda quadrática pode ser apropriada. Por exemplo, em muitos problemas de estatística para o qual a perda simétrica em $(\theta-a)$ é conveniente, a exata forma funcional não é decisiva para a conclusão. A perda quadrática possivelmente pode ser uma aproximação útil para a perda verdadeira.

- A função de perda quadrática ponderada é definida como:

$$
L(\theta, a)=w(\theta)[\theta-a]^{2} .
$$

Esta função tem características atrativas, devido a sua simplicidade e pelo fato de ser ponderada por uma função de $\theta$.

- A função da perda quadrática generalizada é definida como:

$$
L(\Theta, a)=(\Theta-a)^{t} Q(\Theta-a),
$$

onde $\Theta=\left(\theta_{1}, \ldots, \theta_{p}\right)^{t}$ é um vector estimado por $a=\left(a_{1}, \ldots, a_{p}\right)^{t}$ e $\mathbf{Q}_{P \times p}$ é uma matriz não negativa. Quando $Q$ é diagonal a expressão (1.6) se reduz a :

$$
L(\theta, a)=\sum_{i=1}^{p} q_{i}\left(\theta_{i}-a_{i}\right)^{2},
$$

que é uma extensão natural da perda quadrática para situações multivariáveis. 


\subsubsection{Função de Perda Linear}

Quando a função de utilidade é aproximadamente linear, a função de perda tende a ser linear, sendo definida como:

$$
L(\theta, a)= \begin{cases}K_{0}(\theta-a) & \text { se } \theta-a \geq 0, \\ K_{1}(a-\theta) & \text { se } \theta-a<0 .\end{cases}
$$

As constantes $K_{0}$ e $K_{1}$ podem ser escolhidas para refletir a importância de subestimar e superestimar, onde as mesmas usualmente são diferentes.

Quando elas são iguais, a função de perda é equivalente a função de perda do erro absoluto, que é dado por:

$$
L(\theta, a)=|\theta-a|
$$

Se $K_{0}$ e $K_{1}$ são funções de $\theta$, a perda se comporta como uma função linear ponderada. A função de perda linear (ou perda linear ponderada) é uma boa aproximação para encontrar uma perda verdadeira.

\subsubsection{Função de Perda " 0 - 1 "}

Os problemas de decisão de duas ações, por exemplo testes de hipótese, são caracterizados da seguinte forma $a_{0}$ é "correto" se $\theta \in \Theta_{0}$, e $a_{1}$ é correto se $\theta \in \Theta_{1}$, ou seja, corresponde a testar $H_{0}=\theta \in \Theta_{0}$ versus $H_{1}=\theta \in \Theta_{1}$. A função de perda "0 - 1" para este caso é definida como:

$$
L\left(\theta, a_{i}\right)=\left\{\begin{array}{ll}
0 & \text { se } \theta \in \Theta_{i}, \\
1 & \text { se } \theta \in \Theta_{j},
\end{array} \quad(j \neq i) .\right.
$$

A perda é 0 se é tomada uma decisão correta, e 1 se é tomada uma decisão incorreta. $O$ interesse desta perda surge, quando estamos interessados na função do risco associado a esta regra de decisão simples, $\delta(x)$. Logo, $R(\theta, \delta)=E_{\theta}[L(\theta, \delta(x))]=P_{\theta}(\delta(x))$ é a probabilidade de uma decisão incorreta, ou, a probabilidade do erro tipo I ou tipo II dependendo se $\theta \in \Theta_{0}$ ou $\theta \in \Theta_{1}$. 
Na prática, a função de perda " 0-1 " é raramente uma boa aproximação para a perda verdadeira. Portanto, podemos definir funções de perdas mais realistas que serão:

$$
L\left(\theta, a_{i}\right)=\left\{\begin{array}{ll}
0 & \text { se } \theta \in \Theta_{i}, \\
K_{i} & \text { se } \theta \in \Theta_{\mathrm{j}},
\end{array} \quad(j \neq i),\right.
$$

e

$$
L\left(\theta, a_{i}\right)=\left\{\begin{array}{ll}
0 & \text { se } \theta \in \Theta_{i}, \\
K_{i}(\theta) & \text { se } \theta \in \Theta_{\mathrm{j}},
\end{array} \quad(j \neq i) .\right.
$$

Notamos que somente duas funções de perda, $L\left(\theta, a_{0}\right)$ e $L\left(\theta, a_{1}\right)$ são determinadas para as expressões enunciadas acima.

\subsection{Teoria da Decisão Bayesiana}

$\mathrm{Na}$ seção (1.2), foi abordado a teoria clássica das decisões. Um ponto de vista alternativo é a teoria da decisão Bayesiana ou "Subjetiva" exposta por Savage (1954), Lindley (1971), DeGroot (1970), Berger (1985), onde o conhecimento a priori do experimento tem um papel importante. As duas teorias são apropriadas em um conjunto de situações especiais, e cada uma delas possui limitações.

A seguir descreveremos dois pontos importantes no nosso estudo.

1. A teoria clássica é fundamentalmente empírica, porque somente utiliza informação amostral para estimar e fazer provas; enquanto o enfoque Bayesiano utiliza todas as informações disponíveis, baseando-se no juízo pessoal como evidência empírica.

2. A estatística Bayesiana pode ser classificada em duas categorias diferentes que estão estreitamente relacionadas. Primeiramente, temos o que se denomina Inferência Bayesiana Pura, a qual se relaciona com a estimação e comprovação mediante informação subjetiva ou empírica sem considerar "funções de perdas" formais associadas a decisões incorretas. A segunda categoria é conhecida como Teoria da Decisão Bayesiana, a qual envolve princípios e procedimentos para escolher uma ação ótima em uma dada situação, baseando-se em algumas ou todas as informações de perdas, formalmente expressas e explicitamente avaliadas através de funções. 
Basicamente, a inferência Bayesiana consiste em usar a informação a priori e a amostra. O termo "informação a priori" consiste na informação que o estatístico possui sobre um problema antes de efetuar qualquer amostragem. Para incluir tal informação na análise, devemos expressá-la em termos probabilísticos, visto que a informação a priori consiste em juízos pessoais da situação. Os Bayesianos seguem a interpretação subjetiva da probabilidade, que representa "grau de incerteza" em distintas proposições. Porém, eles aceitam as suposições de população normal, variância conhecida, que também são usuais na inferência clássica.

O método Bayesiano, pode ser considerado realmente como uma extensão do enfoque clássico. Como veremos em determinadas situações, ambos métodos conduzirão as mesmas conclusões através de diferentes interpretações.

A seguir, faremos uma introdução sobre as densidades a priori que são utilizadas no decorrer desta dissertação, introduziremos definições de função de risco e risco de Bayes e analisaremos algumas outras funções de perdas encontradas na literatura.

\subsection{Formulação do Teorema de Bayes}

A decisão Bayesiana combina o conhecimento a priori de $\theta$ com a informação estocástica fornecida pelo experimento, através de uma distribuição a posteriori de $\theta$, e escolhe a ação que minimiza a perda esperada a posteriori.

Este procedimento é caracterizado pelo Teorema de Bayes, que é dado da seguinte forma:

$$
p(\theta \mid x)=\frac{p(x \mid \theta) \pi(\theta)}{p_{x}(x)}=\frac{p(x \mid \theta) \pi(\theta)}{\int p(x \mid \theta) \pi(\theta) d \theta}
$$

onde

$p(\theta \mid x)$ : é a densidade a posteriori.

$p(x \mid \theta)$ : é a verossimilhança da observação condicionada ao parâmetro.

$\pi(\theta)$ : é a densidade a priori. Interpreta-se também como o conhecimento que o estatístico adquiriu antes de realizar o experimento.

$p_{x}(x)$ : é definido como a densidade marginal das observações. 
Exemplo 2. ( Ilustração da teoria clássica e Bayesiana )

Uma certa companhia petrolifera, deve tomar uma decisão com respeito a uma área que ainda não foi perfurada e tem alta probabilidade de ter petróleo.

A seguir temos os seguintes estados da natureza.

$$
\Theta=\left\{\begin{array}{l}
\theta_{1}: \text { tem petróleo } \\
\theta_{2}: \text { não tem petróleo }
\end{array}\right.
$$

As ações possiveis da companhia são:

$$
A=\left\{\begin{array}{l}
a_{1}: \text { perfurar } \\
a_{2}: \text { Vender parte do campo a outra companhia } \\
a_{3}: \text { Vender o campo a outra companhia }
\end{array}\right.
$$

A companhia decidiu realizar uma medição geológica para verificar se certa formação geológica está presente ou não.

O espaço amostral consta de dois pontos:

$$
X= \begin{cases}0 & \text { formação geológica ausente } \\ 1 & \text { formação geológica presente }\end{cases}
$$

A presença ou ausência de petróleo afeta a probabilidade da presença ou ausência de formação geológica de acordo com a seguinte função de verossimilhança:

$p(x \mid \theta): \quad$\begin{tabular}{|c|c|c|}
\hline & \multicolumn{2}{|c|}{$x$} \\
\cline { 2 - 3 }$\theta$ & 0 & 1 \\
\hline$\theta_{1}$ & 0.3 & 0.7 \\
\hline$\theta_{2}$ & 0.6 & 0.4 \\
\hline
\end{tabular}


O experimento resume a informação a respeito de $\theta$ através da função de verossimilhança .

Suponha que o estatístico, antes de observar $x$, está disposto a afirmar que áreas similares a área de interesse apresentam uma probabilidade a priori de $3 / 4$ de existir petróleo (isto é feito com base em seu conhecimento técnico). Portanto tem-se que:

$$
\pi\left(\theta_{i}\right): \begin{array}{|l|l|}
\hline \theta & \pi(\theta) \\
\hline \theta_{1} & 0.75 \\
\hline \theta_{2} & 0.25 \\
\hline
\end{array}
$$

Finalmente, o estatístico, avalia sua perda monetária das diferentes ações frente aos possíveis estados da natureza.

Suponhamos a seguinte função de perda $L(\theta, a)$ :

$L(\theta, a): \quad$\begin{tabular}{|l|l|l|l|}
\hline \multirow{2}{*}{} & \multicolumn{3}{|c|}{$a$} \\
\cline { 2 - 4 } & $a_{1}$ & $a_{2}$ & $a_{3}$ \\
\hline$\theta_{1}$ & -10 & 0 & -5 \\
\cline { 2 - 4 }$\theta_{2}$ & 2 & -9 & -4 \\
\hline
\end{tabular}

Agora o problema está completamente especificado. O estatístico deve tomar uma decisão que minimize sua perda.

Por enquanto, a perda do estatístico depende totalmente do estado da natureza $\theta$, o qual é desconhecido antes de tomar sua decisão. Por este motivo, necessitamos do estudo de uma teoria de decisões sob incerteza que desenvolveremos no presente capitulo. 
A primeira tarefa de um estatístico é tentar obter um quadro coerente do problema, como através dos enunciados e tabelas apresentados no exemplo 2. Este é um problema estatístico, com apenas uma situação de escolha: o estatístico deve decidir entre as alternativas $a_{1}, a_{2}, a_{3}$, onde apenas uma deve ser escolhida, e quando isto é feito o problema está resolvido. Porém, na vida real, as pessoas devem tomar uma série de decisões em diferentes instantes; além dos problemas serem dinâmicos, uma decisão leva a outra, e assim por diante. Mais ainda, os problemas são interrelacionados, ou seja, as possíveis ações e estados posteriores podem depender de decisões tomadas anteriormente. $\grave{A}$ medida que o problema se torna mais complexo, pode ser muito dificil especificar e quantificar as relações entre os eventos incertos e as alternativas possíveis. Essas consequências de problemas de decisão são chamadas problemas decisão em multi-estágio, sendo modelados e analisados pelas chamadas árvores de decisão.

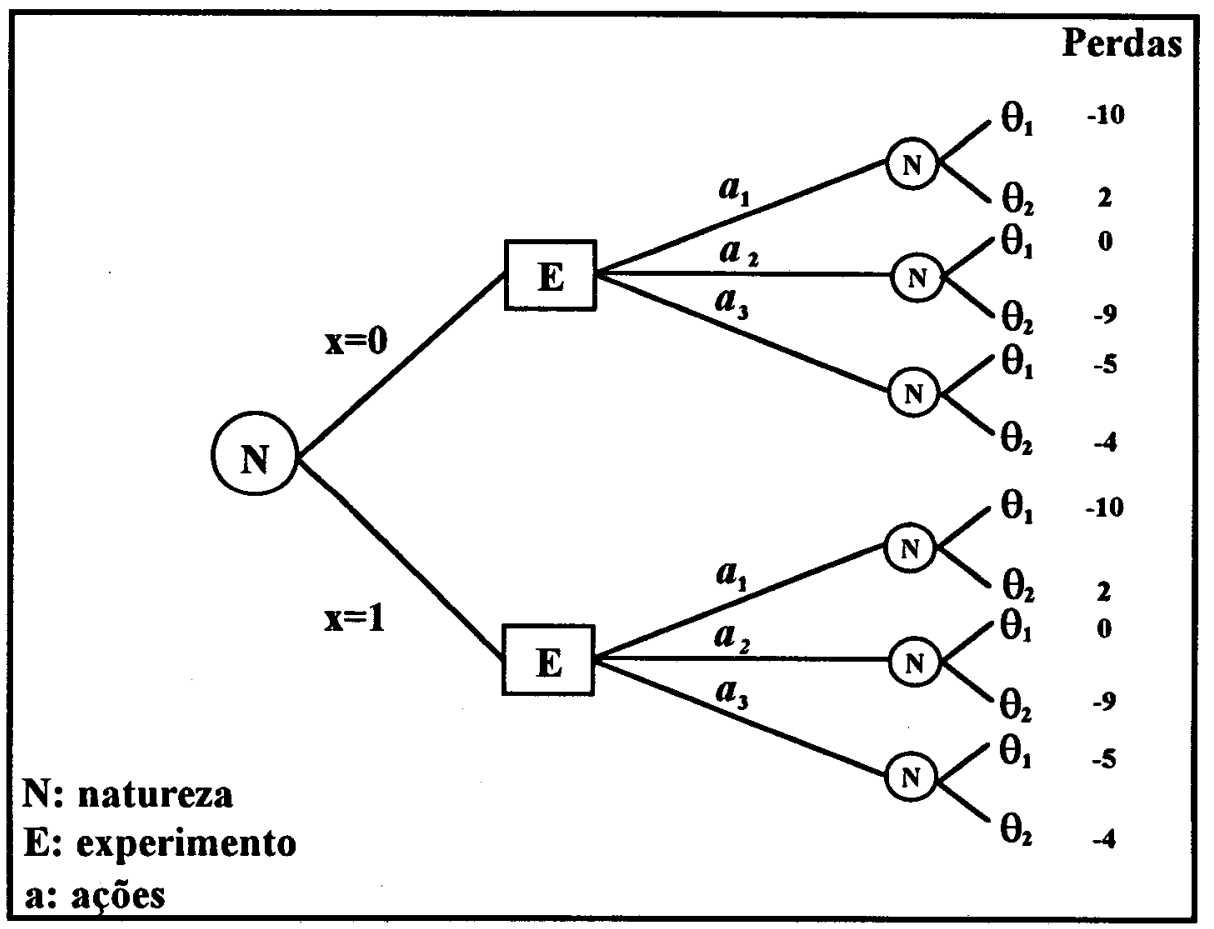

Figura 1.1 A representação do exemplo 2. como um árvore de decisão 
Desta forma, as chamadas árvores de decisão podem ser usadas para determinar decisões ótimas porque capacitam o estatístico a decompor um problema complexo em inúmeros problemas menores. Da figura 1.1, observa-se que os círculos e quadrados são chamados nós ou pontos de decisão, e cada ramo da árvore que se origina destes pontos, representa uma alternativa (ação) $a_{1}, a_{2}, a_{3}$. No fim de cada ramo de ação há um círculo chamado nó o ponto de acaso, no qual o ramo se subdivide em $n$ outros ramos, um para cada estado da natureza possível.

Normalmente, coloca-se sobre os ramos que partem dos nós de acaso as probabilidades de ocorrência dos eventos. No fim destes, podem estar outros nós de decisão, de acaso ou as consequências (resultados) finais que em nosso caso serão dado pelas diferentes perdas.

\section{Exemplo 3. ( continuação do exemplo 2 )}

Utilizando o exemplo 2 e as definições da estatística clássica mostraremos as possiveis combinações das regras de decisão da seguinte maneira:

$$
\begin{array}{ll}
X=\{0,1\} & A=\left\{a_{1}, a_{2}, a_{3}\right\} \\
\delta_{1}(0)=a_{1} & \\
\delta_{2}(0)=a_{1} & \delta_{1}(1)=a_{1} \\
\delta_{3}(0)=a_{1} & \delta_{2}(1)=a_{2} \\
\delta_{4}(0)=a_{2} & \delta_{3}(1)=a_{3} \\
\delta_{5}(0)=a_{2} & \delta_{4}(1)=a_{1} \\
\delta_{6}(0)=a_{2} & \delta_{5}(1)=a_{2} \\
\delta_{7}(0)=a_{3} & \delta_{6}(1)=a_{3} \\
\delta_{8}(0)=a_{3} & \delta_{7}(1)=a_{1} \\
\delta_{9}(0)=a_{3} & \delta_{8}(1)=a_{2} \\
& \delta_{9}(1)=a_{3}
\end{array}
$$


Tabela 1.1 Combinações Possíveis das Regras de Decisão

\begin{tabular}{|c|c|c|c|c|c|c|c|c|c|}
\hline \multirow{2}{*}{$X$} & \multicolumn{10}{|c|}{$\delta$} \\
\cline { 2 - 10 } & $\delta_{1}$ & $\delta_{2}$ & $\delta_{3}$ & $\delta_{4}$ & $\delta_{5}$ & $\delta_{6}$ & $\delta_{7}$ & $\delta_{8}$ & $\delta_{9}$ \\
\hline 0 & $a_{1}$ & $a_{1}$ & $a_{1}$ & $a_{2}$ & $a_{2}$ & $a_{2}$ & $a_{3}$ & $a_{3}$ & $a_{3}$ \\
\hline 1 & $a_{1}$ & $a_{2}$ & $a_{3}$ & $a_{1}$ & $a_{2}$ & $a_{3}$ & $a_{1}$ & $a_{2}$ & $a_{3}$ \\
\hline
\end{tabular}

Uma vez distribuídas as regras de decisão e usando a definição do risco, obteremos os seguintes resultados em termos risco e das diferentes regras de decisão, que serão mostrados na seguinte tabela 1.2 .

A obtenção do risco e a regra de decisão $\delta_{2}$ :

$$
\begin{aligned}
R(\theta, \delta) & =L\left(a_{1}, \theta\right) P_{x \mid \theta}\left(\delta(x)=a_{1}\right)+L\left(a_{2}, \theta\right) P_{x \mid \theta}\left(\delta(x)=a_{2}\right)+L\left(a_{3}, \theta\right) P_{x \mid \theta}\left(\delta(x)=a_{3}\right), \\
R\left(\theta_{2}, \delta_{2}\right) & =L\left(a_{1}, \theta_{1}\right) P_{x \mid \theta}\left(\delta_{2}(x)=a_{1}\right)+L\left(a_{2}, \theta_{2}\right) P_{x \mid \theta}\left(\delta_{2}(x)=a_{2}\right)+L\left(a_{3}, \theta_{3}\right) P_{x \mid \theta}\left(\delta_{2}(x)=a_{3}\right) \\
& =(2) P\left(x=0 / \theta_{2}\right)+(-9) P\left(x=1 / \theta_{1}\right)+(-4)(0), \\
& =-2.4
\end{aligned}
$$

Tabela 1.2 Resultados do Risco, quando se Usa a Regra de Decisão $\delta(x)$, e o Estado da Natureza $\theta$.

\begin{tabular}{|c|c|c|c|c|c|c|c|c|c|}
\hline \multirow{2}{*}{$R$} & \multicolumn{10}{|c|}{$\delta$} \\
\cline { 2 - 10 } & $\delta_{1}$ & $\delta_{2}$ & $\delta_{3}$ & $\delta_{4}$ & $\delta_{5}$ & $\delta_{6}$ & $\delta_{7}$ & $\delta_{8}$ & $\delta_{9}$ \\
\hline$R\left(\theta_{1}, \delta\right)$ & -10 & -3 & -6.5 & -7.0 & 0.0 & -3.5 & -8.5 & -1.5 & -5.0 \\
\hline$R\left(\theta_{2}, \delta\right)$ & 2 & -2.4 & -0.4 & -4.6 & -9.0 & -7.0 & -1.6 & 6.0 & -4.0 \\
\hline
\end{tabular}

Desta tabela podemos analisar que o risco dado por $R\left(\theta_{1}, \delta_{1}\right)=-10$, representa a perda média mínima quando o estatístico utiliza a regra de decisão $\delta_{1}(x)$ e o estado da natureza $\theta_{1}$. Similarmente observamos que temos uma perda média mínima quando se usa $R\left(\theta_{2}, \delta_{5}\right)=-9.0$ e frente a estes dois resultados podemos optar por utilizar $R\left(\theta_{1}, \delta_{1}\right)$, por ter menor perda. 


\subsection{Elementos Básicos na Teoria da Decisão Bayesiana.}

Como a teoria de decisão Bayesiana é extensão da teoria clássica, podemos usar todos seus elementos, sendo adicionada a estes a distribuição de probabilidade $\pi(\theta)$, onde o conhecimento do estatístico é subjetiva.

Nesta seção, introduzimos certas definições especificas a serem utilizadas neste trabalho, as quais supoem a existência de conhecimento a priori dado pela densidade a priori e uma função de perda $L(\theta, \delta)$.

Definição 1.8.1 O risco de Bayes para uma regra de decisão $\delta$ é definido como o valor esperado do risco $R(\theta, \delta)$ em relação a distribuição a priori $\pi(\theta)$, isto é:

$$
r(\pi, \delta)=E[R(\theta, \delta)]=\int_{\theta} \int_{x} L(\theta, \delta(x)) f(x \mid \theta) \pi(\theta) d x d \theta
$$

Definição 1.8.2 A regra de decisão $\delta_{0}$, é uma regra de decisão ótima para a distribuição a priori $\pi$, se e somente se:

$$
r(\pi)=r\left(\pi, \delta_{\pi}\right)=\min _{\delta \in \mathrm{D}} r(\pi, \delta),
$$

onde $r(\pi)$ é conhecida como o risco de Bayes mínimo em relação a $\pi$.

Definição 1.8.3 A perda Bayesiana esperada a posteriori de $\delta(x)$ em relação a distribuição a priori $\pi(\theta)$ é definida por:

$$
\rho(\pi, \delta(x))=E_{\theta \mid x}[L(\theta, \delta(x))]=\int_{\theta} L(\theta, \delta(x)) \pi(\theta \mid x) d \theta
$$

Definição 1.8.4 $A$ ação $\delta^{*}(x)$ é uma ação ótima condicionada em $x$, para a distribuição a posteriori, se e somente se:

$$
\rho\left(\pi, \delta^{*}(x)\right)=\operatorname{mim}_{\delta \in D} \rho(\pi, \delta(x))
$$


Está definição também é conhecida como "Princípio de minimização de perda esperada". Portanto, para cada $x$, escolheremos a ação $\delta^{*}(x)$, tal que a perda seja mínima.

\section{Exemplo 4. (continuação do exemplo 2)}

A partir da tabela de riscos 1.2 , e das probabilidades a priori $\pi\left(\theta_{1}\right)=3 / 4 \mathrm{e}$ $\pi\left(\theta_{2}\right)=1 / 4$, podemos fazer os cálculos e encontrar os riscos Bayesianos médios.

Tabela 1.3 Resultados dos Riscos Bayesianos.

\begin{tabular}{|l|l|l|l|l|l|l|l|l|l|}
\hline$\delta$ & $\delta_{1}$ & $\delta_{2}$ & $\delta_{3}$ & $\delta_{4}$ & $\delta_{5}$ & $\delta_{6}$ & $\delta_{7}$ & $\delta_{8}$ & $\delta_{9}$ \\
\hline$r(\pi, \delta)$ & -7 & -2.85 & -4.98 & -6.4 & -2.25 & -4.38 & -6.78 & -2.63 & -4.75 \\
\hline
\end{tabular}

Da tabela 1.3, observamos que a melhor decisão será $\delta_{1}$, ou seja tomando a ação $a_{1}$, quando $x=0$ ou $x=1$.

Para obter a melhor ação de Bayes condicional, calculamos as probabilidades a posteriori segundo a regra de Bayes, ou seja:

$$
\begin{aligned}
& P\left(\theta_{1} \mid x=0\right) \propto P\left(x=0 \mid \theta_{1}\right) \pi\left(\theta_{1}\right)=(0.3)(0.5)=0.25 \\
& P\left(\theta_{2} \mid x=0\right) \propto P\left(x=0 \mid \theta_{2}\right) \pi\left(\theta_{2}\right)=(0.6)(0.25)=0.15 \\
& P\left(\theta_{1} \mid x=1\right) \propto P\left(x=1 \mid \theta_{1}\right) \pi\left(\theta_{1}\right)=(0.7)(0.75)=0.525 \\
& P\left(\theta_{2} \mid x=1\right) \propto P\left(x=1 \mid \theta_{2}\right) \pi\left(\theta_{2}\right)=(0.4)(0.25)=0.10
\end{aligned}
$$

Normalizando tanto para $x=0$, como $x=1$, obtemos:

$$
\begin{array}{ll}
P\left(\theta_{1} \mid x=0\right)=0.64 & P\left(\theta_{1} \mid x=1\right)=0.84 \\
P\left(\theta_{2} \mid x=0\right)=0.4 & P\left(\theta_{2} \mid x=1\right)=0.16
\end{array}
$$


As perdas esperadas a posteriori para $x=0$ e $a_{1}, a_{2}, a_{3}$ é dado por:

$$
\begin{aligned}
\rho\left(\pi, a_{1}\right) & =R\left(\theta_{1}, \delta_{1}(x)\right) P\left(\theta_{1} \mid x=0\right)+R\left(\theta_{2}, \delta_{1}(x)\right) P\left(\theta_{2} \mid x=0\right), \\
& =(-10)(0.6)+(2)(0.4)=-5, \\
\rho\left(\pi, a_{2}\right) & =-3.6, \\
\rho\left(\pi, a_{3}\right) & =-4.6 .
\end{aligned}
$$

Portanto, a melhor decisão condicionada em $x=0$ é dada pela ação $a_{1}$.

As perdas esperadas a posteriori para $x=1$ e $a_{1}, a_{2}, a_{3}$ são dadas por:

$$
\begin{aligned}
\rho\left(\pi, a_{1}\right) & =R\left(\theta_{1}, \delta_{1}(x)\right) P\left(\theta_{1} \mid x=1\right)+R\left(\theta_{2}, \delta_{1}(x)\right) P\left(\theta_{2} \mid x=1\right), \\
& =(-10)(0.84)+(2)(0.16)=-8.08, \\
\rho\left(\pi, a_{2}\right) & =-1.44, \\
\rho\left(\pi, a_{3}\right) & =-4.84 .
\end{aligned}
$$

Portanto, a melhor decisão condicionada em $x=1$ é dada pela ação $a_{1}$. Finalmente, concluimos que $\delta_{1}$ é a melhor regra de decisão do conjunto das decisões.

\subsubsection{Densidades a Priori}

Uma vantagem do uso dos métodos Bayesianos na análise de dados é que podemos introduzir na análise a informação a priori sobre os parâmetros. No caso de não ter informação sobre os parâmetros, o grau de conhecimento deles é representado através de uma densidade a priori não-informativa.

As densidades a priori são classificadas, em densidades a priori "informativas" e "nãoinformativas". Utiliza-se as densidades a priori informativas quando o pesquisador dispõe de informação sobre o parâmetro, proveniente se sua experiência sobre o modelo, suas considerações teóricas ou das informações contidas em amostras de dados passados. Algumas densidades a priori, tanto não-informativas, como informativas são consideradas nas seções subsequentes.

$\mathrm{Na}$ literatura Bayesiana existem inúmeras densidades a priori não-informativas, as mais conhecidas são priori de Jeffreys (1967) e Zellner (1984) . 


\subsection{Funções de Perdas na Teoria de Decisão Bayesiana}

A utilização das funções de perdas, nos proporciona numerosas simplificações nos problemas de estimação. Do ponto de vista Bayesiano, toda informação (subjetiva e experimental) está resumida na distribuição a posteriori de $\theta$, onde o estimador de Bayes é uma função dos dados que minimiza a perda média a posteriori. Este estimador Bayesiano para $\theta$ será denotado por:

$$
\hat{\theta}=\delta(x),
$$

onde $x$ é observado e $\delta(x)$ é uma regra de decisão.

A seguir faremos uma apresentação de algumas funções de perdas conhecidas na literatura e o resumo de suas propriedades são apresentadas nas tabelas (1.4) e (1.5), (ver Martz e Waller 1986 ).

\section{- Função de Perda Quadrática}

A função de perda quadrática, devido a sua simplicidade matemática é amplamente usada em estimação estatística.

Definição 1.9.1 A funcão de perda quadrática é definida por:

$$
L(\theta, \hat{\theta})=a(\theta-\hat{\theta})^{2}, \quad \theta \in \Theta
$$

Definição 1.9.2 A função de risco da função da perda quadrática é definida por:

$$
R(\theta, \hat{\theta})=a E\left[(\theta-\hat{\theta})^{2} \mid \theta\right]
$$

onde $E\left[(\theta-\hat{\theta})^{2} \mid \theta\right]$ é o erro quadrático médio. 
Tabela 1.4 Algumas Propriedades do Estimador de Bayes para uma Função de Perda Quadrática $L(\theta, \hat{\theta})=a(\theta-\hat{\theta})^{2}, \quad \mathrm{a}>0$.

\begin{tabular}{|c|l|l|}
\hline Propriedade & \multicolumn{1}{|c|}{ Valor } & \multicolumn{1}{c|}{ Explicação } \\
\hline Risco & $a \int_{x}[\theta-E(\theta \mid x)]^{2} f(x \mid \theta) d x$ & \\
\hline Risco a posteriori & $a \operatorname{Var}(\Theta \mid x)$ & Proporcional a variância a posteriori \\
\hline Risco de Bayes & $a E_{x}\left[\operatorname{var}_{\theta \mid x}(\Theta \mid x)\right]$ & $\begin{array}{l}\text { Proporcional a esperança da variância } \\
\text { a posteriori }\end{array}$ \\
\hline Média & $E(\Theta)$ & Média a priori \\
\hline Variância & $\operatorname{Var}(\Theta)-E_{x}\left[\operatorname{Var}_{\theta \mid x}(\Theta \mid x)\right]$ & $\begin{array}{l}\text { Diferença entre a variância a priori e a } \\
\text { esperança da variância a posteriori }\end{array}$ \\
\hline
\end{tabular}

Definição 1.9.3 A função de perda quadrática generalizada é definida como:

$$
L(\Theta, \hat{\Theta})=(\Theta-\hat{\Theta})^{t} A(\Theta-\hat{\Theta})
$$

onde $\Theta=\left(\theta_{1}, \ldots, \theta_{p}\right)^{t}$ com $p \geq 2$, sendo estimado por $\hat{\Theta}=\left(\theta_{1}, \ldots, \theta_{p}\right)^{t}$ e $A_{p \times p}$ uma matriz simétrica não negativa.

Suponhamos que o vetor médio $E(\Theta \mid \mathbf{x})$ e a matriz de $\operatorname{covariância~} \operatorname{Cov}(\Theta \mid \mathbf{x})$ da distribuição a posteriori de $\Theta$ dado x exista.

O estimador que minimiza o risco é obtido da seguinte expressão:

$$
\begin{aligned}
E\left[(\Theta-\hat{\Theta})^{t} A(\Theta-\hat{\Theta}) \mid \mathbf{x}\right]= & E\left\{[\Theta-E(\Theta \mid \mathbf{x})]^{t} A[\Theta-E(\Theta \mid \mathbf{x})] \mid \mathbf{x}\right\}+[E(\Theta \mid \mathbf{x})-\hat{\Theta}]^{t} A[E(\Theta \mid \mathbf{x})-\hat{\Theta}] \\
& =\operatorname{traço}[A \operatorname{Cov}(\Theta \mid \mathbf{x})]+[E(\Theta \mid \mathbf{x})-\hat{\Theta}]^{t} A[E(\Theta \mid \mathbf{x})-\hat{\Theta}]
\end{aligned}
$$

Minimizando o risco (1.20) em $\hat{\Theta}$ obtemos: 


$$
\hat{\Theta}=E(\Theta \mid \mathbf{x})=\int_{\Theta} \Theta \pi(\Theta \mid \mathbf{x}) d \Theta
$$

Assim, a média a posteriori $\hat{\Theta}=E(\Theta \mid \mathbf{x})$ é o estimador de Bayes para $\Theta$.

Tabela 1.5 Algumas Propriedades do Estimador de Bayes para uma Função de Quadrática Generalizada $L(\Theta, \hat{\Theta})=(\Theta-\hat{\Theta})^{t} A(\Theta-\hat{\Theta})$.

\begin{tabular}{|c|l|l|}
\hline Propriedade & \multicolumn{1}{|c|}{ Valor } & \multicolumn{1}{|c|}{ Explicação } \\
\hline Risco & $\int_{x}[\Theta-E(\Theta \mid \mathbf{x})]^{t} A[\Theta-E(\Theta \mid \mathbf{x})] f(\mathbf{x} \mid \Theta) d \Theta$ & \\
\hline Risco a posteriori & $\operatorname{traço}\left\{A\left[\operatorname{cov}_{\Theta \mid x}(\Theta \mid \mathbf{x})\right]\right\}$ & $\begin{array}{l}\text { Traço do produto de A e a matriz de } \\
\text { covariância a posteriori. }\end{array}$ \\
\hline Risco de Bayes & $\operatorname{traço}\left\{A E_{x}\left[\operatorname{cov}_{\Theta \mid x}(\Theta \mid \mathbf{x})\right]\right\}$ & $\begin{array}{l}\text { Traço do produto de A e esperança da } \\
\text { matriz de covariância a posteriori. }\end{array}$ \\
\hline Média & $E(\Theta)$ & Vector de médias a priori. \\
\hline Covariância & $\operatorname{cov}(\Theta)-E_{x}\left[\operatorname{cov}_{\Theta \mid x}(\Theta \mid \mathbf{x})\right]$ & $\begin{array}{l}\text { Diferença entre a matriz de } \\
\text { covariância a priori e a esperança da } \\
\text { matriz de covariância a posteriori. }\end{array}$ \\
\hline
\end{tabular}

\section{- Função de Perda do Erro-absoluto}

Definição 1.9.4 A função de perda do erro- absoluto é definida como:

$$
L(\theta, \hat{\theta})=a|\theta-\hat{\theta}|, \quad a>0 .
$$

Assume-se que a perda é proporcional ao valor absoluto do erro de estimação. $\mathrm{O}$ estimador de Bayes é aquele que minimiza o risco a posteriori dado por $E(a|\Theta-\hat{\theta}| \mathbf{x})$.

Chernoff e Moses (1959, pág. 319) mostraram que o valor de $\hat{\theta}$ que minimiza o risco a posteriori é a mediana da distribuição a posteriori de $\Theta$ dado $\mathbf{x}$, se $P_{r}(\Theta \geq \hat{\theta} \mid \mathbf{x}) \geq 0.5$ e 
$P_{r}(\Theta \leq \hat{\theta} \mid \mathbf{x}) \geq 0.5$. O estimador que satisfaz as desigualdades acima é conhecido como a mediana a posteriori.

O risco de Bayes é dado pela solução da seguinte equação:

$$
r(\pi)=a E_{\mathbf{x}}\left[E_{\theta \mid \mathbf{x}}(|\Theta-\hat{\theta}| \mathbf{x})\right]
$$

onde $\hat{\theta}$ é a mediana da distribuição a posteriori de $\Theta$ dado $\mathbf{x}$.

\section{- Outras Funções de Perdas}

A seguir apresentaremos outras funções de perdas, muitos usadas em problemas Econométricos (ver Zellner, Journal Econometrics, 37, 1988).

Definição 1.9.5 A função de perda quadrática relativa é dada por:

$$
L(\theta, \hat{\theta})=\frac{(\theta-\hat{\theta})^{2}}{\theta^{2}}
$$

onde $\hat{\theta}$ é o estimador de $\theta$.

Seja $\theta=\frac{1}{\beta}$, o interesse é obter informação sobre $\beta$ através da distribuição a posteriori, a qual está baseada sobre uma densidade a priori informativa ou não-informativa para $\beta$. A expressão (1.24) pode ser reformulada como:

$$
L(\theta, \hat{\theta})=(1-\hat{\theta} \beta)^{2}
$$

Desenvolvendo o quadrado em (1.25) e aplicando valor esperado obtemos:

$$
E L(\theta, \hat{\theta})=1-2 \hat{\theta} E(\beta)+\hat{\theta}^{2} E\left(\beta^{2}\right)
$$


O estimador pontual Bayesiano é obtido derivando em relação a $\hat{\theta}$ a expressão (1.26):

$$
\hat{\theta}^{*}=\frac{E(\beta)}{E\left(\beta^{2}\right)}=\frac{1}{E(\beta)} \times \frac{1}{1+\operatorname{var}(\beta) /[E(\beta)]^{2}},
$$

onde $E(\beta)$ e $\operatorname{var}(\beta)$ são a média e a variância a posteriori de $\beta$, respectivamente.

Definição 1.9.6 A função de perda quadrática generalizada é dada por:

$$
L(\theta, \hat{\theta})=\beta_{2}^{2}(\theta-\hat{\theta})^{2},
$$

onde $\hat{\theta}$ é o estimador de $\theta$.

Seja $\theta=\frac{\beta_{1}}{\beta_{2}}$, o interesse é comparar $\beta_{1}$ e $\beta_{2}$ através de uma distribuição a posteriori, a qual está baseada sobre uma densidade a priori informativa ou não-informativa para $\beta_{1}$ e $\beta_{2}$. A expressão (1.28) pode ser reformulada como:

$$
L(\theta, \hat{\theta})=\left(\beta_{1}-\hat{\theta} \beta_{2}\right)^{2} .
$$

Desenvolvendo o quadrado em (1.29), e aplicando valor esperado obtemos:

$$
E L(\theta, \hat{\theta})=E\left(\beta_{1}^{2}\right)-2 \hat{\theta} E\left(\beta_{1} \beta_{2}\right)+\hat{\theta}^{2} E\left(\beta_{2}^{2}\right) .
$$

O estimador pontual Bayesiano é obtido derivando em relação a $\hat{\theta}$ a expressão (1.30):

$$
\hat{\theta}^{*}=\frac{E\left(\beta_{1} \beta_{2}\right)}{E\left(\beta_{2}^{2}\right)}=\frac{E\left(\beta_{1}\right)}{E\left(\beta_{2}\right)}\left(\frac{1+\operatorname{cov}\left(\beta_{1} \beta_{2}\right) / E\left(\beta_{1}\right) E\left(\beta_{2}\right)}{1+\operatorname{var}(\beta) /\left[E\left(\beta_{2}\right)\right]^{2}}\right),
$$

onde a $\operatorname{cov}\left(\beta_{1}, \beta_{2}\right)$ e $\operatorname{var}\left(\beta_{2}\right)$ denota a covariância a posteriori de $\beta_{1}$ e $\beta_{2}$ e a variância de $\beta_{2}$, respectivamente. 


\section{CAPÍTULO 2}

\section{ESTIMAÇÃO DO PARÂMETRO DE UMA DISTRIBUIÇÃO EXPONENCIAL}

\subsection{Introdução}

Neste capítulo, apresentaremos a forma de obter estimadores não viciados para o parâmetro da distribuição exponencial utilizando algumas funções de perdas. Estes estimadores também serão obtidos através da análise Bayesiana e finalizando, faremos uma comparação entre as duas classes.

\subsection{Estimadores Clássicos}

Suponhamos que temos uma amostra aleatória de tamanho $n, \mathbf{x}=\left\{x_{1}, \ldots, x_{n}\right\}$, retirada de uma distribuição exponencial dada por:

$$
f(x \mid \theta)=\theta e^{-\theta x}, \quad(x \geq 0, \theta>0),
$$

onde $\theta$ é um parâmetro desconhecido. Nosso objetivo é estimar $\theta$ usando os dados da amostra. Para estimar $\theta$, El-Sayyad (1967) propôs um estimador da forma $\frac{d_{n}}{S}$, onde $S=\sum x_{i}$ é uma estatística suficiente para $\theta$ e $d_{n}$ é uma função de $n$.

Estimativas pontuais, da forma proposta por "El-Sayyad", serão obtidas usando conceitos de função de perda. Para isto, seja $x$ uma amostra aleatória e $g(x)$ uma estimativa para $\theta$. Dada uma função de perda $L(g(x), \theta)$, onde $\theta$ é o valor do parâmetro, o risco associado coma estimativa $g(x)$ é definido como: 


$$
R_{g}(\theta)=E[L(g(x), \theta)] .
$$

Usando o conceito do risco, Lehmann (1951) forneceu a seguinte definição "Uma estimativa $g(x)$ é não viciada em relação a uma função de perda $L(g(x), \theta)$, se para cada $\theta, E\left[L\left(g(x), \theta_{0} \mid \theta\right)\right]$ é mínimo quando $\theta_{0}=\theta$ ". Por sua vez, Goodman (1953) usou esta definição e derivou alguns estimadores correspondentes à funções de perdas diferentes.

Seguindo as mesmas ideias de Goodman, alguns resultados gerais serão obtidos e utilizados para calcular $d_{n}$.

Suponhamos que $\mathrm{T}$ é uma v.a., cuja distribuição depende de um parâmetro $\theta$ desconhecido.

Consideremos a seguinte função de perda:

$$
L(g, \theta)=\lambda(\theta)\left(g^{\beta}-\theta^{\beta}\right)^{2},
$$

onde $\beta$ é uma constante conhecida e $\lambda(\theta)$ é uma função positiva de $\theta$.

Lema 1. Suponhamos que A seja uma constante conhecida satifazendo a seguinte expressão:

$$
\theta^{\beta} E\left(T^{-\beta} \mid \theta\right)=A E\left(T^{-2 \beta} \mid \theta\right), \quad(2 \beta \leq n) .
$$

Então, entre todos os estimadores da forma $d / T$, o risco

$$
R_{d}(\theta)=E\left[\lambda(\theta)\left\{(d / T)^{\beta}-\theta^{\beta}\right\}\right],
$$

é minimizando quando $d=A^{1 / \beta}$.

Demonstração: seja,

$$
R_{d}(\theta)=\lambda(\theta) E\left[(d / T)^{\beta}-\theta^{\beta}\right]^{2} .
$$

Desenvolvendo o quadrado e tomando o valor esperado em $T$ obtemos:

$$
R_{d}(\theta)=\lambda(\theta) E\left[d^{2 \beta} E\left(T^{-2 \beta} \mid \theta\right)-2 d^{\beta} A E\left(T^{-2 \beta} \mid \theta\right)+\theta^{2 \beta}\right],
$$




$$
\begin{aligned}
\frac{\partial R_{d}(\theta)}{\partial d^{\beta}} & =2 \lambda(\theta) E\left(T^{-2 \beta} \mid \theta\right)\left(d^{\beta}-a\right)=0, \\
d & =A^{1 / \beta} .
\end{aligned}
$$

Se tomarmos $\beta=1, y=\frac{1}{T}$ e usando Lema 1 , obtemos o seguinte resultado: Seja $A$ uma constante conhecida tal que:

$$
\theta E(y \mid \theta)=A E\left(y^{2} \mid \theta\right)
$$

então, $A y$ é o estimador que minimiza o risco dado por:

$$
R_{d}(\theta)=E\left\{\lambda(\theta)(d y-\theta)^{2} \mid \theta\right\}
$$

Lema 2. Suponhamos que (2.4) seja satisfeita entre todos os estimadores da forma $g=\frac{d}{T}$, o único estimador não viciado em relação a função de perda

$$
L(g, \theta)=\theta^{-2 \beta}\left(g^{\beta}-\theta^{\beta}\right)^{2},
$$

é dado por $T^{-1} A^{1 / \beta}$.

Demonstração: Seja,

$$
\begin{aligned}
& R_{d}(\theta)=E\left[\theta^{-2 \beta}\left\{(d / T)^{\beta}-\theta^{\beta}\right\}^{2}\right] \mathrm{e} \\
& R_{d}\left(\theta_{0}\right)=E\left[\theta_{0}^{-2 \beta}\left\{(d / T)^{\beta}-\theta_{0}\right\}^{2}\right] .
\end{aligned}
$$

Desenvolvendo o quadrado de $R_{d}\left(\theta_{0}\right)$ e usando (2.3) obtemos:

$$
R_{d}\left(\theta_{0}\right)=1+d^{2 \beta} \theta_{0}^{-2 \beta} E\left(T^{-2 \beta} \mid \theta\right)-2 A d^{\beta}\left(\theta \theta_{0}\right)^{\beta} E\left(T^{-2 \beta} \mid \theta\right) .
$$

A expressão (2.6) é uma função quadrática de $\theta_{0}^{-\beta} \mathrm{e}$ 


$$
\frac{\partial R_{d}\left(\theta_{0}\right)}{\partial \theta_{0}^{-\beta}}=-2 \theta_{0}^{-\beta} E\left(T^{-2 \beta} \mid \theta\right)+2 A d^{\beta}\left(\theta^{-2 \beta}\right) E\left(T^{-2 \beta} \mid \theta\right)=0,
$$

se e somente se

$$
\frac{A}{\theta^{\beta}}=\left(\frac{d}{\theta_{0}}\right)^{\beta} \text {. }
$$

Consequentemente, $R_{0}(\theta)$ é minimizada para todo $\theta_{0}=\theta$, se somente se:

$$
d=A^{1 / \beta} \text {. }
$$

Consideremos agora outra função de perda dada por:

$$
L(g, \theta)=\lambda(\theta)(\log g-\log \theta)^{2} .
$$

Seja $T$ uma v.a. cuja distribuição depende de um parâmetro desconhecido e $\lambda(\theta)$ é uma função positiva de $\theta$.

Lema 3. Suponhamos que:

$$
E\{\log \theta T \mid \theta\}=A
$$

onde A é conhecida entre todos os estimadores da forma $d / T$, sendo d constante. Então, o risco é dado por:

$$
R_{d}(\theta)=E\left[(\lambda(\theta))\left\{\log d T^{-1}-\log \theta\right\}^{2} \mid \theta\right]
$$

o qual é minimizado quando $d=\exp (A)$.

Demonstração: Seja,

$$
\begin{gathered}
R_{d}(\theta)=\lambda(\theta) E\left\{\left(\log d T^{-1}\right)^{2}-2 \log d T^{-1} \log \theta+(\log \theta)^{2} \mid \theta\right\}, \\
\frac{\partial R_{d}(\theta)}{\partial \log d}=\lambda(\theta) E\{(2 \log d-\log T)-2 \log \theta\}=0 .
\end{gathered}
$$

Desenvolvendo a equação (2.9), obtemos:

$$
d=\exp (A)
$$


Lema 4. Suponha que (2.8) seja satisfeita, então entre todos os estimadores da forma $\boldsymbol{g}=d / T$, o único estimador não viciado com respeito a função de perda

$$
L(g, \theta)=(\log g-\log \theta)^{2}
$$

é dado por $e^{A} / T$.

Este estimador, pelo Lema 3 é o que minimiza o risco. A demonstração deste Lema é similar a demonstração dos Lemas 1 e 2.

\section{Exemplo 2.1}

Seja $\mathbf{x}=\left\{x_{1}, \ldots, x_{n}\right\}$ uma amostra aleatória de tamanho $n$, de uma distribuição exponencial com função de probabilidade dada por:

$$
f(x \mid \theta)=\theta e^{-\theta x}, \quad(x \geq 0, \theta>0)
$$

A função de verossimilhança é dada por:

$$
\ell(\mathbf{x} \mid \theta)=\theta^{n} e^{-\theta S}
$$

onde $S=\sum x_{i}$. Sabemos que $2 \theta S$ tem uma distribuição qui-quadradado com $2 n$ graus de liberdade. Portanto, a densidade de S é dada por:

$$
f(S \mid \theta)=\frac{\theta^{n} S^{n-1} e^{-\theta S}}{\Gamma(n)}
$$

onde $\Gamma(n)$ é a função gama.

Consideremos a seguinte função de perda:

$$
L_{1}(g, \theta)=\lambda(\theta)\left\{(d / S)^{\beta}-\theta^{\beta}\right\}
$$

onde $g=d / S$. Pelo Lema 1, temos: 


$$
A=\frac{\theta E\left(T^{-\beta} \mid \theta\right)}{E\left(T^{-2 \beta} \mid \theta\right)} .
$$

Da expressão (2.12) obtemos:

$$
\begin{aligned}
E\left(T^{-\beta} \mid \theta\right) & =\int_{0}^{\infty} \frac{T^{-\beta} \theta^{n} T^{(n-1)} e^{-\theta T}}{\Gamma(n)} d T, \\
& =\frac{\Gamma(n-\beta)}{\theta^{-\beta} \Gamma(n)} \int_{0}^{\infty} \frac{T^{(n-\beta)} \theta^{(n-\beta)} e^{-\theta T}}{\Gamma(n-\beta)} d T,
\end{aligned}
$$

fazendo as devidas simplificações em (2.13), obtemos:

$$
E\left(T^{-\beta} \mid \theta\right)=\frac{\Gamma(n-\beta)}{\theta^{-\beta} \Gamma(n)}
$$

Analogamente,

$$
\begin{aligned}
E\left(T^{-2 \beta} \mid \theta\right) & =\int_{0}^{\infty} \frac{T^{-2 \beta} \theta^{(N-2 \beta)} T^{(n-1)} e^{-\theta T}}{\Gamma(n)} d T \\
& =\frac{\Gamma(n-2 \beta)}{\theta^{-2 \beta} \Gamma(n)} \int_{0}^{\infty} \frac{T^{(n-2 \beta-1)} \theta^{(n-2 \beta)} e^{-\theta T}}{\Gamma(n-2 \beta)} d T, *
\end{aligned}
$$

simplificando (2.15), obtemos:

$$
E\left(T^{-2 \beta} \mid \theta\right)=\frac{\Gamma(n-2 \beta)}{\theta^{-2 \beta} \Gamma(n)}
$$

Sustituindo (2.14) e (2.16) em (2.12), obtemos:

$$
A=\frac{\Gamma(n-\beta)}{\Gamma(n-2 \beta)}
$$

* Apêndice A 
portanto,

$$
g_{1}(x \mid \theta)=S^{-1}\left\{\frac{\Gamma(n-\beta)}{\Gamma(n-2 \beta)}\right\}^{1 / \beta}
$$

Pelo Lema $2, g_{1}$ é um estimador não viciado em relação a função de perda.

$$
L_{1}(g, \theta)=\lambda(\theta)\left\{(d / S)^{\beta}-\theta^{\beta}\right\}^{2},
$$

onde $\lambda(\theta)=\theta^{-2 \beta}$.

Consideremos o caso especial quando $\beta=1$. Portanto de (2.18), obtemos:

$$
g_{1}=S^{-1}\left\{\frac{\Gamma(n-1)}{\Gamma(n-2)}\right\}=\frac{n-2}{S}
$$

Pelo Lema 1, $g_{1}=\frac{(n-2)}{S}$, é um estimador de máxima verossimilhança não viciado e com menor erro quadrático dado por:

$$
\begin{gathered}
\operatorname{var}\left(\frac{n-2}{S}\right)=\left(\frac{n-2}{n-1}\right)^{2} K, \text { pois, } \\
(n-2)^{2} \operatorname{var}\left(\frac{1}{S}\right)=(n-2)^{2}\left\{\frac{\theta^{2}}{(n-1)(n-2)}\right\}=\left(\frac{n-2}{n-1}\right)^{2} K
\end{gathered}
$$

Consideremos agora a seguinte função de perda:

$$
L_{2}(g, \theta)=\lambda(\theta)\{\log (d / S)-\log \theta\}^{2} .
$$

Tomando $g_{1}=\frac{d}{S}$ e $E(\log \theta S)=A$ ( $A$ conhecida), temos pelo Lema 3 que o estimador que mínimiza o risco associado à função de perda (2.19) é dado por $g_{2}=e^{A} / S$. Porém, como:

$$
A=\frac{\theta^{n}}{\Gamma(n)} \int_{0}^{\infty} \log (\theta S) S^{n-1} e^{-\theta S} d S
$$


temos:

$$
A=\frac{\partial}{\partial n} \log \Gamma(n)
$$

Definindo,

$$
\phi(n)=\exp \left\{\left(\frac{\partial}{\partial n}\right), \log \Gamma(n)\right\}
$$

obtemos:

$$
g_{2}=\frac{\phi(n)}{S}
$$

Pelo Lema $4, g_{2}$ é um estimador não viciado com relação à função de perda (2.19), e com $\lambda(\theta)=1$, tambem, foi considerado por Girshick (1951) como um estimador mínimax admissivel.

\subsection{Estimadores Bayesianos}

O objetivo desta seção é obter estimativas do ponto de vista Bayesiano usando algumas funções de perdas.

Seja, $\mathbf{x}=\left(x_{1}, \ldots, x_{n}\right)$ uma amostra, $g(\mathbf{x})$ uma estimativa para $\theta$ e $L(g, h)$ uma função de perda correspondente, onde $h=h(\theta)$.

Dado $\mathbf{x}$, a perda esperada a posteriori do estimador $g(\mathbf{x})$ de $h(\theta)$ é dada por:

$$
\int L(g, h) p(\theta \mid x) d \theta
$$

O estimador Bayesiano para $h$ é definido como uma função $g(\mathbf{x})$ que minimiza (2.20). Suponhamos que (2.20) exista e que as condições de regularidade para diferenciar sob o sinal de integração estejam satisfeitas, então o estimador Bayesiano é dado como uma solução da seguinte equação:

$$
\int \frac{\partial L(g, h)}{\partial g} p(\theta \mid x) d \theta=0
$$




\section{Exemplo 2.2 (continuação do exemplo 2.1)}

Muitas vezes o Bayesiano enfrenta o problema de escolher uma priori apropriada que possa combinar facilmente com sua verossimilhança (2.9). Este problema foi solucionado quando Barnard (1954) introduziu as famílias conjugadas que foram usadas por Raiffa e Schlaifer (1961). A vantagem das famílias conjugadas é que a posteriori pertenece a mesma famílias das densidades a priori.

Para o nosso exemplo consideramos uma priori conjugada dada por:

$$
\pi(\theta) \propto \theta^{a-1} e^{-b \theta}, \quad(\theta \geq 0) \quad \forall a, b>0 .
$$

Combinando (2.9) com (2.22) obtemos a distribuição a posteriori dada por:

$$
p(\theta \mid x) \propto \theta^{a+n-1} e^{-(b+s) \theta}
$$

Portanto:

$$
\begin{gathered}
p(\theta \mid S)=\frac{\theta^{a+n-1} e^{-(b+S) \theta}}{\int \pi(\theta) p(S \mid \theta) d \theta}, \\
=\frac{\theta^{a+n-1} e^{-(b+S) \theta}}{\frac{\Gamma(a+n)}{(b+S)} \int_{0}^{\infty} \frac{(b+S)^{a+n} \theta^{a+n-1} e^{-(b+S) \theta}}{\Gamma(a+n)} d \theta},
\end{gathered}
$$

fazendo as devidas simplificações em (2.23), obtemos:

$$
p(\theta \mid x)=\frac{(b+S)^{a+n} \theta^{a+n-1} e^{-(b+s) \theta}}{\Gamma(n)}
$$

Consideremos a seguinte função de perda:

$$
L_{3}(g, \theta)=\theta^{\alpha}\left(g^{\beta}-\theta^{\beta}\right)^{2},
$$

onde $\lambda(\theta)=\theta^{\alpha}$.

\footnotetext{
* ver apêndice $A$
} 
Usando (2.21), o estimador de Bayes satisfaz a seguinte expressão:

$$
g_{3}^{\beta}=\frac{E\left(\theta^{\alpha+\beta} \mid S\right)}{E\left(\theta^{\alpha} \mid S\right)} .
$$

Desenvolvendo o numerador de (2.25) obtemos,

$$
\begin{aligned}
E\left(\theta^{\alpha+\beta} \mid S\right) & =\int_{0}^{\infty} \frac{\theta^{\alpha+\beta}(b+S)^{a+n} \theta^{a+n-1} e^{-(b+S) \theta}}{\Gamma(a+n)} d \theta \\
& =\frac{1}{\Gamma(a+n)} \int_{0}^{\infty} \theta^{\alpha+\beta+n-1}(b+S)^{a+n} e^{-(S+b) \theta} d \theta \\
& =\frac{\Gamma(\alpha+\beta+a+n)}{(b+S)^{\alpha+\beta-1} \theta \Gamma(a+n)} \int_{0}^{\infty} \frac{\theta^{\alpha+\beta+a+n}(b+S)^{(\alpha+\beta+a+n)-1} e^{-(S+b) \theta}}{\Gamma(\alpha+\beta+a+n)} d \theta,{ }^{*}
\end{aligned}
$$

simplificando (2.26), obtemos:

$$
E\left(\theta^{\alpha+\beta} \mid S\right)=\frac{\Gamma(\alpha+\beta+a+n)}{(b+S)^{\alpha+\beta-1} \theta \Gamma(a+n)} .
$$

Analogamente,

$$
\begin{aligned}
E\left(\theta^{\alpha} \mid S\right) & =\int_{0}^{\infty} \frac{\theta^{\alpha}(b+S)^{a+n} \theta^{a+n-1} e^{-(b+s) \theta}}{\Gamma(a+n)} d \theta, \\
& =\frac{1}{\Gamma(a+n)} \int_{0}^{\infty} \theta^{\alpha+a+n-1}(b+S)^{a+n} e^{-(S+b) \theta} d \theta, \\
& =\frac{\Gamma(\alpha+a+n)}{(b+S)^{\alpha-1} \theta \Gamma(a+n)} \int_{0}^{\infty} \frac{\theta^{\alpha+a+n}(b+S)^{(\alpha+a+n)-1} e^{-(S+b) \theta}}{\Gamma(\alpha+a+n)} d \theta,
\end{aligned}
$$

simplificando (2.28), obtemos:

\footnotetext{
* ver apêndice $A$
} 


$$
E\left(\theta^{\alpha} \mid S\right)=\frac{\Gamma(\alpha+a+n)}{(b+S)^{\alpha-1} \theta \Gamma(a+n)} .
$$

Substituindo (2.27) e (2.29) em (2.25), obtemos:

$$
g_{3}^{\beta}=(b+S)^{-\beta}\left\{\frac{\Gamma(\alpha+\beta+a+n)}{\Gamma(\alpha+a+n)}\right\} .
$$

Assim, o estimador Bayesiano é dado por,

$$
g_{3}=(b+S)^{-1}\left\{\frac{\Gamma(\alpha+\beta+a+n)}{\Gamma(\alpha+a+n)}\right\}^{1 / \beta}
$$

Analogamente, consideremos a função de perda:

$$
L_{4}(g, \theta)=(\log g-\log \theta)^{2},
$$

fazendo os cálculos, o estimador Bayesiano é dado por: $g_{4}=\phi(a+n) /(b+S)$.

\subsection{Comparação entre o Método Clássico e o Bayesiano}

Para comparar o método clássico com o Bayesiano foi preciso utilizar alguns lemas e diferentes funções de perdas na obtenção dos estimadores, com seus correspondentes riscos.

O maior problema que enfrenta o Bayesiano é a escolha de sua priori (informação passada sobre $\theta$ ). Existem vários procedimentos que nos conduzem a obter densidades a priori não-informativas. Essa diversidade de métodos para desenvolver densidades a priori não-informativas concerne a dificuldade de como expressar a noção de "pouca informação". 
A maioria dos métodos começa com a tentativa de medir a quantidade de informação contida na densidade a priori, através da comparação de densidades a priori com a função de verossimilhança ou através do grau de influência que a densidade a priori causa na posteriori.

Para o exemplo apresentado neste capítulo, foi utilizado uma densidade a priori nãoinformativa também chamada de priori de Jeffreys (1967), $\pi(\theta) \sim \frac{1}{\theta}$, a qual foi obtida do argumento baseado na invariância sob transformações e na medida de informação do Fisher.

Os estimadores clássicos foram obtidos para diferentes funções de perdas. Usando a função de perda (2.11) e o Lema 2, e obtivemos:

$$
g_{1}=S^{-1}\left\{\frac{\Gamma(n-\beta)}{\Gamma(n-2 \beta)}\right\}^{1 / \beta}
$$

onde $g_{1}$ é um estimador não viciado para $\lambda(\theta)=\theta^{-2 \beta}$. Na abordagem Bayesiana para $\lambda(\theta)=\theta^{\alpha}$, usando uma função de perda $L_{3}(g, \theta)$, obtivemos o estimador dado por:

$$
g_{3}=(b+S)^{-1}\left\{\frac{\Gamma(\alpha+\beta+a+n)}{\Gamma(\alpha+a+n)}\right\}^{1 / \beta}
$$

Para um estudo comparativo tomamos $a=b=0$ e $\alpha=-2 \beta$, obtivemos os seguintes resultados:

$$
g_{1}=g_{3} \text { e } g_{2}=g_{4},
$$

Os dois métodos tanto clássico como Bayesiano produzem o mesmo estimador, pois a priori utilizada no método Bayesiano é não informativa.

A seguir apresentaremos uma tabela, com algumas funções de perdas para o numerador $d_{n}$, do estimador $d_{n} / S$. 
Tabela 2.1. Algumas Funções de Perda para o Numerador $d_{n}$.

\begin{tabular}{|c|c|c|}
\hline $\mathrm{N}^{\circ}$ & $d_{n}$ & Função de Perda \\
\hline 1 & $\sim n-1$ & $\{\sqrt[3]{g}-\sqrt[3]{\theta}\}^{2} / \theta^{2 / 3}$ \\
\hline 2 & $\sim n-5 / 4$ & $\{\sqrt{g}-\sqrt{\theta}\} / \theta$ \\
\hline 3 & $=n-2$ & $(g-\theta)^{2} / \theta^{2}$ \\
\hline 4 & $\sim n-1 / 2$ & $(\log g-\log \theta)^{2}$ \\
\hline
\end{tabular}

Da Tabela 2.1, observamos:

- Todos os estimadores de $\theta$ têm variância menor que o estimador de máxima verossimilhança.

- O estimador (1) é uma aproximação para o estimador não viciado de $\theta$.

- O estimador (3) tem variância e erro quadrático médio menor que o estimador de máxima verossimilhança e é não viciado para $\theta$.

- O estimador (4) é minimax admíssivel. 


\section{CAPÍtULO 3}

\section{ESTIMAÇÃO BAYESIANA NA CONFIABILIDADE USANDO UMA FUNÇÃO DE PERDA ASSIMÉTRICA}

\subsection{Introdução}

Neste capítulo, obteremos uma estimativa Bayesiana de confiabilidade para o modelo exponencial utilizando uma densidade a priori e uma função de perda assimétrica.

A função de perda definida neste capítulo foi introduzida por Canfield (1970), como uma função que possibilita ponderar a perda em relação à superestimação. Em outras palavras, a perda associada com a superestimação é geralmente maior do que a perda associada com a subestimação.

Para obter uma estimativa da confiabilidade, é utilizada uma aproximação Bayesiana baseada no nível de significancia inferior. (ver exemplo de Martz e Waller, 1982).

\subsection{Formulação do Modelo Exponencial}

Uma análise Bayesiana será desenvolvida assumindo que os tempos de falha dos componentes têm distribuição exponencial com função densidade dada por:

$$
f(t, \theta)=\frac{1}{\theta} e^{-t / \theta}, \quad \mathrm{t} \geq 0
$$

onde $\theta$ é a taxa de falha .

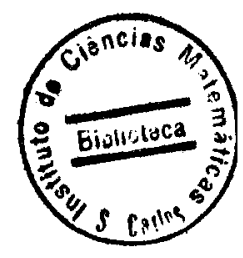


Nos ensaios de confiabilidade ou situações práticas de teste de vida, os dados de sobrevivência, em geral, são incompletos ou censurados. Isto pode ocorrer devido ao próprio planejamento do experimento considerado para a coleta de dados.

Um plano amostral muito usado nesta área é dado pelo esquema de censuras de tipo II, onde sob as condicões ambientais, o teste é aplicado nos componentes e termina quando um número pré-determinado de falhas é obtido.

Um outro esquema de censura é dado pelo esquema de censura de tipo I. Neste esquema, são observados os tempos de sobrevivência de $\boldsymbol{n}$ unidades durante um tempo fixo $t$. Este plano amostral tem grande aplicação na área médica. Lawless (1982) descreve outros planos.

Suponhamos que temos uma amostra de tamanho $n$, onde as $r$ primeiras observações $t_{(1)}<t_{(2)}<, \ldots,<t_{(r)}$ são avaliadas e sua densidade conjunta é dada por:

$$
\ell\left(t_{1}, \ldots, t_{r} \mid \theta\right)=\frac{n !}{(n-r) !} \times \frac{1}{\theta} \exp \left[-\left(\sum_{i=1}^{r} t_{(i)}+(n-r) t_{(r)}\right) / \theta\right]
$$

onde, $T=\sum_{i=1}^{r} t_{(i)}+(n-r) t_{(r)}$ representa o tempo total de um certo equipamento testado e $r$ é o número de falhas observadas. $O$ tempo total $T$ tem uma distribuição dada por $\frac{2 T}{\theta} \sim \chi_{(2 \mathrm{r})}^{2}$.

A flexibilidade da função da verossimilhança permite o desenvolvimento da densidade a posteriori e a obtenção do estimador pontual Bayesiano através do nivel de significancia inferior os quais são aplicados a uma ampla variedade de problemas sobre teste de vida.

A confiabilidade do modelo exponencial é definida como:

$$
R=R(\theta, t)=e^{-\theta t},
$$

onde $\theta$ é a taxa de falha, e $t$ é o tempo fixado. 


\subsection{Função de Perda Assimétrica}

Nesta seção, usaremos uma função de perda a qual nos permite medir o grau de assimétria que será formulada através dos níveis de confiança usuais $R_{\alpha}$ de $R(\theta, t)$ que é dado por:

$$
R_{\alpha}=R\left(\theta_{\alpha}, t\right)=e^{-\theta_{\alpha} t}
$$

onde

$$
\begin{gathered}
2 T \theta_{\alpha}=\chi_{v, \alpha}^{2}, \\
F_{\chi_{v}^{2}}\left(2 T \theta_{\alpha}\right)=1-\alpha \mathrm{e}
\end{gathered}
$$

$\theta_{\alpha}$ é um estimador de $\theta$ ou $R_{\alpha}$ é um estimador da confiabilidade $R$. O parâmetro $v$ tem o valor $2 r$ no caso de censura tipo $I I$, e $2 r+2$ no caso de censura tipo $I$.

Sejam as seguintes funções de perdas definidos em termos da confiabilidade de $R$.

$$
\begin{aligned}
& L_{1}\left(\theta_{\alpha}, \theta\right)=\frac{1}{\theta}\left(\frac{R_{\alpha}}{R}-1\right)^{2} \mathrm{e} \\
& L_{2}\left(\theta_{\alpha}, \theta\right)=\frac{2 \gamma}{\theta}\left(\frac{R_{\alpha}}{R}-1\right) .
\end{aligned}
$$

A função de perda assimétrica proposta por Canfield (1970) é dada por:

$$
L\left(\theta_{\alpha}, \theta\right)= \begin{cases}L_{1}\left(\theta_{\alpha}, \theta\right) & \text { se } \theta_{\alpha} \geq \theta \\ L_{1}\left(\theta_{\alpha}, \theta\right)+L_{2}\left(\theta_{\alpha}, \theta\right) & \operatorname{se} \theta_{\alpha}<\theta\end{cases}
$$

O parâmetro $\gamma$ em (3.6) controla a superestimação $\left(\theta_{\alpha} \geq \theta\right)$ na função de perda, e quando $\gamma=0$, a função de perda $L\left(\theta_{\alpha}, \theta\right)$ em (3.7) é a função de perda quadrática (simétrica). 
A seguir apresentaremos a figura 3.1 o gráfico da função de perda assimétrica com valores diferentes para $\gamma$.

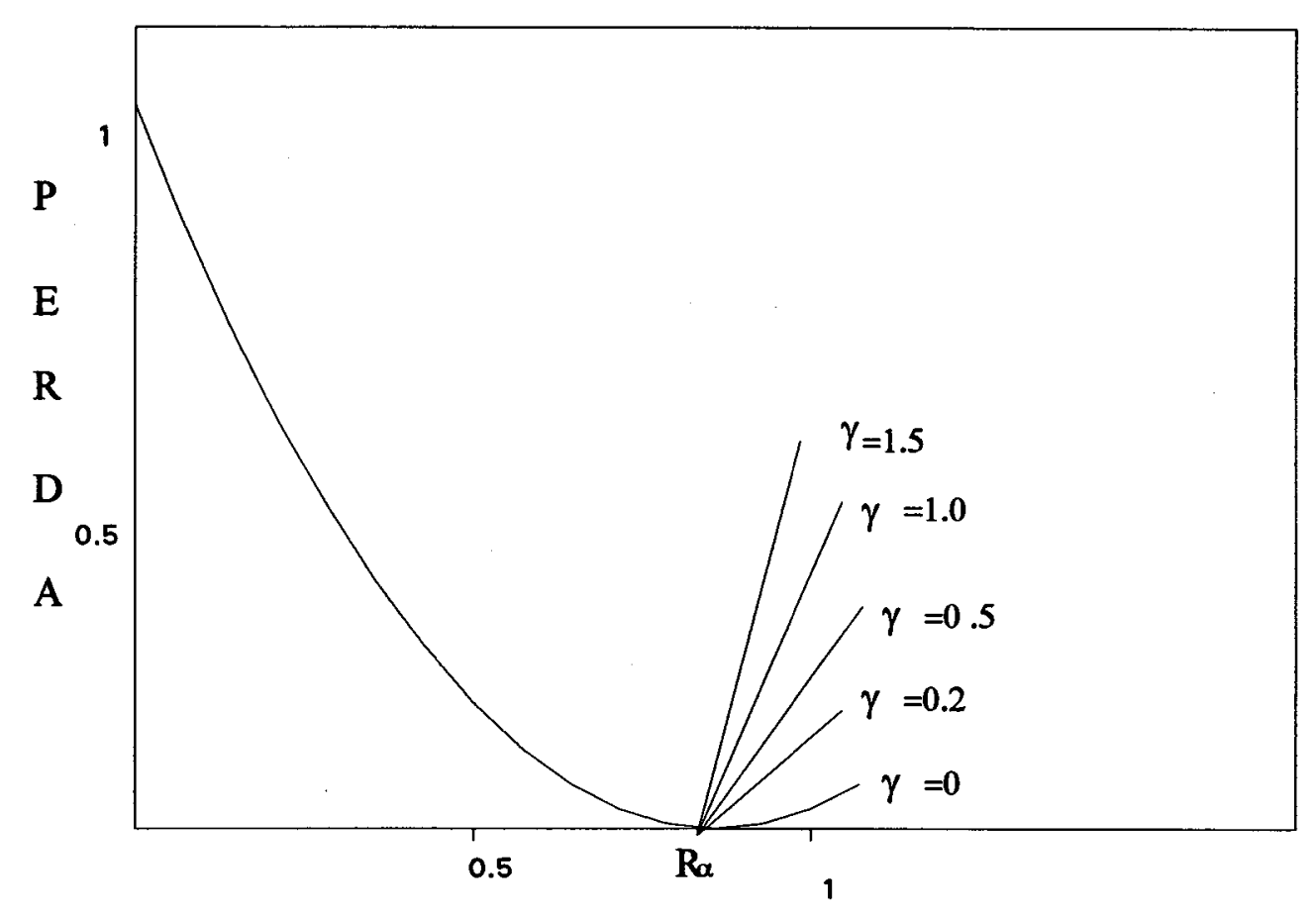

Fig 3.1 Gráfico da função de perda

\section{Exemplo 3.1}

Se a confiabilidade é fixada em 0.9 e subestimada em 0.0625 , para diferentes valores de $\gamma$, temos:

Para $\gamma=0 \Rightarrow R_{\alpha}=0.8375$ e a perda é de 0.0048 .

Para $\gamma=1 \Rightarrow R_{\alpha}=0.9625$ e a perda é de 0.187 .

Para $\gamma=1.5 \Rightarrow R_{\alpha}=0.9625$ e a perda é de 0.275 .

Assim, quando aumentamos o valor de $\gamma$, produz-se uma perda maior para superestimação quando é comparado com a subestimação. 


\subsection{Densidade a Priori}

Muitas vezes, supõe-se que o pesquisador não tem conhecimento a priori da distribuição do parâmetro R. O problema do estatístico Bayesiano consiste em encontrar uma densidade a priori conveniente, dado o espaço paramétrico de seu parâmetro. Neste caso, é considerada uma densidade a priori muita usada na confiabilidade com esquema de censura tipo II. Está densidade a priori é dada em termos de uma distribuição Beta e sua função de densidade é dada por:

$$
f(R)=\frac{1}{\beta(p, q)} R^{p-1}(1-R)^{q-1}, \quad 0<R<1
$$

Como $R$ é decrescente em $t$, em geral é difícil formular uma priori para $R$ que tenha está característica, por este motivo é mais conveniente usar uma densidade a priori sobre a taxa de falhas para evitar esta propriedade.

Assim, para determinar uma priori para $\theta, \operatorname{com} t$ convenientemente fixado, faremos uso de (3.4) e das técnicas de transformação de variáveis.

Seja $g(\theta)$ uma priori para $\theta$ e $t=t_{0}$ em (3.4), logo:

$$
g(\theta)=f(R)\left|\frac{d R}{d \theta}\right|
$$

Portanto,

$$
g(\theta)=\frac{1}{\beta(p, q)}\left(e^{-\theta t_{0}}\right)^{p}\left(1-e^{-\theta t_{0}}\right)^{q-1}
$$

Fazendo, $p=0$ e $q=1$, obtemos uma a priori uniforme em $\theta$. 


\subsection{Densidade a Posteriori}

Uma densidade a posteriori, será obtida para $\theta$ usando a densidade a priori (3.9) e dados com censura tipo II. Sabemos que, dado $\theta, 2 \theta T$ tem uma distribuição qui-quadrado $\left(\chi_{2 r}^{2}\right)$ com $2 r$ graus de liberdade e por tanto a densidade condicional de $T$ dado $\theta$ é dado por:

$$
h(T \mid \theta)=\frac{\theta^{r}}{\Gamma(r)} T^{r-1} e^{-\theta T} .
$$

A densidade a posteriori $h(\theta \mid T)$ de $\theta$ é dada por:

$$
\begin{aligned}
h(\theta \mid T) & =K_{1} g(\theta) h(T \mid \theta) \\
& =K_{1} \frac{1}{\beta(p, q)}\left(e^{-\theta t_{0}}\right)^{p}\left(1-e^{-\theta t_{0}}\right)^{q-1} \frac{\theta^{r}}{\Gamma(r)} T^{r-1} e^{-\theta T} .
\end{aligned}
$$

Fazendo as devidas simplificações na expressão acima , temos:

$$
h(\theta \mid T)=K_{2} \theta^{r} e^{-\theta\left(p t_{0}+T\right)}\left[1-e^{-\theta t_{0}}\right]^{q-1},
$$

onde $K_{i}, i=1,2$, é a constante normalizadora, $t$ é o tempo fixo e $t_{0}$ é o tempo usado para a priori de $\theta$.

\subsection{Estimação da Confiabilidade}

Consideremos o risco associado a perda (3.7), isto é,

$$
\begin{aligned}
\beta\left(\theta_{\alpha}\right) & =\int_{0}^{\infty} L\left(\theta_{\alpha}, \theta\right) h(\theta \mid T) d \theta \\
& =\int_{0}^{\infty} L_{1}\left(\theta_{\alpha}, \theta\right) h(\theta \mid T) d \theta+\int_{\theta_{\alpha}}^{\infty} L_{2}\left(\theta_{\alpha}, \theta\right) h(\theta \mid T) d \theta,
\end{aligned}
$$

onde $\theta_{\alpha}$ é o estimador de $\theta$. 
$O$ estimador de Bayes para a confiabilidade de $R$ é obtida para valores de $\alpha$ que minimizam a equação (3.12). Para encontrar o valor de $\theta_{\alpha}$ que minimiza $\beta\left(\theta_{\alpha}\right)$, basta diferenciar as perdas em (3.12) com relação a $\theta_{\alpha}$ como segue:

$$
\beta^{\prime}\left(\theta_{\alpha}\right)=\int_{0}^{\infty} \frac{\partial L_{1}}{\partial \theta_{\alpha}} h(\theta \mid T) d \theta+\int_{\theta_{\alpha}}^{\infty} \frac{\partial L_{2}}{\partial \theta_{\alpha}} h(\theta \mid T) d \theta-L_{2}\left(\theta_{\alpha}, \theta_{\alpha}\right) h\left(\theta_{\alpha} \mid T\right),
$$

onde $L_{2}\left(\theta_{\alpha}, \theta_{\alpha}\right) h\left(\theta_{\alpha} \mid T\right)=0$.

Portanto, $\theta_{\alpha}$ deverá satisfazer a equação:

$$
\beta^{\prime}\left(\theta_{\alpha}\right)=\int_{0}^{\infty} \frac{\partial L_{1}}{\partial \theta_{\alpha}} h(\theta \mid T) d \theta+\int_{\theta_{\alpha}}^{\infty} \frac{\partial L_{2}}{\partial \theta_{\alpha}} h(\theta \mid T) d \theta=0
$$

Para dados com censuras de tipo II, obtemos os seguintes resultados:

$$
\begin{aligned}
& \frac{\partial L_{1}\left(\theta_{\alpha}, \theta\right)}{\partial \theta_{\alpha}}=\frac{-2 t}{\theta}\left(\frac{e^{-\theta_{\alpha} t}}{e^{-\theta t}}-1\right) \frac{e^{-\theta_{\alpha} t}}{e^{-\theta t}}, \\
& \frac{\partial L_{2}\left(\theta_{\alpha}, \theta\right)}{\partial \theta_{\alpha}}=\frac{-2 \gamma t}{\theta}\left(\frac{e^{-\theta_{\alpha} t}}{e^{-\theta t}}\right) .
\end{aligned}
$$

Substituindo (3.14), (3.15) e (3.9) na equação (3.13), obtemos a seguinte expressão:

$$
\begin{aligned}
\beta^{\prime}\left(\theta_{\alpha}\right) & =\int_{0}^{\infty} \frac{-2 t}{\theta}\left(\frac{e^{-\theta_{\alpha} t}}{e^{-\theta t}}-1\right) K_{2} \theta^{r} e^{-\theta\left(p t_{0}+T\right)}\left[1-e^{-\theta t_{0}}\right]^{q-1} d \theta+ \\
& +\int_{\theta_{\alpha}}^{\infty} \frac{-2 \gamma t}{\theta}\left(\frac{e^{-\theta_{\alpha} t}}{e^{-\theta t}}\right) K_{2} \theta^{r} e^{-\theta\left(p t_{0}+T\right)}\left[1-e^{-\theta t_{0}}\right]^{q-1} d \theta
\end{aligned}
$$


Estimação Bayesiana na Confiabilidade Usando uma Função de Perda Assimétrica

44

Colocando $R_{\alpha}$ em evidência em (3.16) e igualando a zero obtemos:

$$
\begin{aligned}
\beta^{\prime}\left(\theta_{\alpha}\right)= & -2 t R_{\alpha}^{2} K_{2} \int_{0}^{\infty} \theta^{r-1} e^{-\theta\left(p t_{0}+T-2 t\right)}\left(1-e^{-\theta t_{0}}\right)^{q-1} d \theta+2 t R_{\alpha} K_{2} \int_{0}^{\infty} \theta^{r-1} e^{-\theta\left(p t_{0}+T-t\right)}\left(1-e^{-\theta t_{0}}\right)^{q-1} d \theta- \\
& -2 \gamma t R_{\alpha} K_{2} \int_{0}^{\infty} \theta^{r-1} e^{-\theta\left(p t_{0}+T-t\right)}\left(1-e^{-\theta t_{0}}\right)^{q-1} d \theta=0 .
\end{aligned}
$$

Fazendo uma expansão binomial da expressão $\left(1-e^{-\theta t_{0}}\right)^{q-1}$ em (3.17), obtemos:

$$
\begin{aligned}
\beta^{\prime}\left(\theta_{\alpha}\right) & =-R_{\alpha}^{2}\left[\sum_{i=1}^{q-1}(-1)^{i}\left(\begin{array}{c}
q-1 \\
i
\end{array}\right) \int_{0}^{\infty} \theta^{r-1} e^{-\left[(p+i) t_{0}+T-2 t\right]} d \theta\right]+R_{\alpha}\left[\sum_{i=1}^{q-1}(-1)^{i}\left(\begin{array}{c}
q-1 \\
i
\end{array}\right) \int_{0}^{\infty} \theta^{r-1} e^{-\theta\left[(p+i) t_{0}+T-t\right]} d \theta\right]- \\
& -\gamma R_{\alpha}\left[\sum_{i=1}^{q-1}(-1)^{i}\left(\begin{array}{c}
q-1 \\
i
\end{array}\right) \int_{\theta_{\alpha}}^{\infty} \theta^{r-1} e^{-\theta\left[(p+i) t_{0}+T-t\right]} d \theta\right]=0
\end{aligned}
$$

Definindo,

$$
(-1)^{i}\left(\begin{array}{c}
q-1 \\
1
\end{array}\right)=w_{i}, \quad \mathrm{e}
$$

colocando $R_{\alpha}$ em evidência em (3.18), obtemos:

$$
R_{\alpha}=\frac{\left[\sum_{i=1}^{q-1} w_{i} \int_{0}^{\infty} \theta^{r-1} e^{-\theta\left[(p+i) t_{0}+T-t\right]} d \theta\right]-\gamma\left[\sum_{i=1}^{q-1} w_{i} \int_{\theta_{\alpha}}^{\infty} \theta^{r-1} e^{-\theta\left[(p+i) t_{0}+T-t\right]} d \theta\right]}{\left[\sum_{i=1}^{q-1} w_{i} \int_{0}^{\infty} \theta^{r-1} e^{-\theta\left[(p+i) t_{0}+T-2 t\right]} d \theta\right]},
$$

ou 


$$
R_{\alpha}=\frac{\left[\sum_{i=1}^{q-1} w_{i} \frac{\Gamma(r)}{\left[(p+i) t_{0}+T-t\right]^{r}}\right]-\gamma\left[\sum_{i=1}^{q-1} w_{i} \int_{\theta_{\alpha}}^{\infty} \theta^{r-1} e^{-\theta\left[(p+i) t_{0}+T-t\right]} d \theta\right]}{\left[\sum_{i=1}^{q-1} w_{i} \frac{\Gamma(r)}{\left[(p+i) t_{0}+T-2 t\right]}\right]}
$$

Fazendo a transformação de variável $x=\theta\left[(p+i) t_{0}+T-t\right]$ na integral em (3.17), obtemos:

$$
\int_{\theta_{\alpha}}^{\infty} \theta^{r-1} e^{-\theta\left[(p+i) t_{0}+T-t\right]} d \theta=\frac{1}{\left[2(p+i) t_{0}+T-t\right]^{r-1}} \int_{x_{0}}^{\infty} x^{r-1} e^{-\frac{x}{2}} \frac{d x}{2\left[(p+i) t_{0}+T-t\right]}
$$

onde

$$
x_{0}=2 \theta_{\alpha}\left[(p+i) t_{0}+T-t\right]
$$

Colocando a integral em termos de uma distribuição qui-quadrado, temos:

$$
\int_{\theta_{\alpha}}^{\infty} \theta^{r-1} e^{-\theta\left[(p+i) t_{0}+T-t\right]} d \theta=\frac{\Gamma(r)}{\left[(p+i) t_{0}+T-t\right]^{r}} \int_{x_{0}}^{\infty} f_{\chi_{2 r}^{2}}(x) d x
$$

onde

$$
\int_{x_{0}}^{\infty} f_{\chi_{2 r}^{2}}(x) d x=\left(1-F_{\chi_{2 r}^{2}}\left(x_{0}\right)\right)=\alpha_{i}^{*}
$$

Substituindo (3.22) em (3.21), obtemos:

$$
\int_{\theta_{\alpha}}^{\infty} \theta^{r-1} e^{-\theta\left[(p+i) t_{0}+T-t\right]} d \theta=\frac{\Gamma(r)}{\left[(p+i) t_{0}+T-t\right]^{r}}\left(\alpha_{i}^{*}\right)
$$

Substituitndo (3.23) em (3.19) e fazendo as devidas simplificações, obtemos: 


$$
R_{\alpha}=\left(1-\frac{t}{T}\right)^{r} \frac{\sum_{i=1}^{q-1} w_{i} A_{i}-\gamma \sum_{i=1}^{q-1} w_{i} A_{i} \alpha_{i}^{*}}{\sum_{i=1}^{q-1} w_{i}\left[1-\frac{t-(p+i) t_{0}}{T-t}\right]^{-r}}
$$

onde

$$
A_{i}=\left[1-\frac{t-(p+i) t_{0}}{T}\right]^{-r}
$$

Dado um $\alpha$ que satisfaça (3.24), então $R_{\alpha}$ dado em (3.24) é o estimador de Bayes da confiabilidade $R$.

Para obter $\alpha$ que satisfaça (3.24), necessitamos calcular $\alpha_{i}^{*}$ que pode ser obtida através da tabela, "Biometrika Tables for Statisticians" por Pearson, E. S. \& Hartley, H.O. (1970).

Para $\gamma=0$ (perda quadrática) a expressão (3.24) se reduz a:

$$
R_{\alpha}=\left(1-\frac{t}{T}\right)^{r}
$$

Quando adotamos uma perda quadrática, o estimador obtido em (3.25) é de variância mínima e não viciado para a confiabilidade.

Usando uma aproximação assintótica em (3.22) obtemos:

$$
\alpha_{i}^{*}=\alpha-\frac{\left[(p+i) t_{0}-t\right]\left(T \theta_{\alpha}\right)^{r} e^{-T \theta_{\alpha}}}{T \Gamma(r)}
$$

Como veremos a seguir, está aproximação é útil na solução (3.24). Para $T$ suficientemente grande (3.24) se reduz a:

$$
\alpha_{i}^{*} \cong \alpha, \forall i
$$

Consequentemente, (3.24) pode ser simplificada obtendo,

$$
R_{\alpha}=(1-\gamma \alpha)\left(1-\frac{t}{T}\right)^{r} \frac{\sum_{i=1}^{q-1}(-1)^{i}\left(\begin{array}{c}
q-1 \\
i
\end{array}\right)\left[1-\frac{t-(p+i) t_{0}}{T}\right]^{-r}}{\sum_{i=1}^{q-1}(-1)^{i}\left(\begin{array}{c}
q-1 \\
i
\end{array}\right)\left[1-\frac{t-(p+i) t_{0}}{T-t}\right]^{-r}}
$$


A confiabilidade para censuras de tipo I é similar a censuras de tipo II. A única diferença é que em (3.24) $r$ é substituido por $(r+1)$ e a distribuição $\chi^{2}$ associada com $R_{\alpha}$ tem $(2 r+2)$ graus de liberdade.

\section{Exemplo 3.2}

A seguir, apresentaremos uma tabela com os resultados das estimativas na confiabilidade, onde dados testados assumiram censura de tipo II, sendo $T$ o tempo acumulado, $r$ o número de falhas e uma densidade a priori em termos de $p$ e $q$.

Tabela 3.1 Estimativas na Confiabilidade

\begin{tabular}{|c|c|c|c|c|c|c|}
\hline $\begin{array}{c}\text { Dados } \\
\text { Testados }\end{array}$ & $\begin{array}{c}\text { Densidade } \\
\text { a priori }\end{array}$ & $\begin{array}{c}95 \% \\
\text { Intervalo de } \\
\text { confiança }\end{array}$ & E.M.V. & $\gamma=0$ & $\gamma=1$ & $\gamma=2$ \\
\hline $\mathrm{T}=50$ & $\mathrm{p}=0$ & & & & & \\
$\mathrm{r}=2$ & $\mathrm{q}=1$ & 0.9095 & 0.96040 & 0.95960 & 0.9152 & 0.9040 \\
$\mathrm{~T}=50$ & $\mathrm{p}=16$ & 0.9095 & 0.96040 & 0.96947 & 0.9162 & 0.9041 \\
$\mathrm{r}=2$ & $\mathrm{q}=1$ & $\mathrm{p}=0$ & & & & \\
\hline $\mathrm{T}=500$ & $\mathrm{p}=1$ & 0.9457 & 0.96075 & 0.96067 & 0.9434 & 0.9423 \\
$\mathrm{r}=20$ & $\mathrm{q}=1$ & & & & \\
\hline
\end{tabular}

Da tabela 3.1, observamos que a coluna (3) apresenta resultados do estimador Bayesiano, quando se usa o nível de significancia inferior com $95 \%$ de confiança, sendo não viciado e de mínima variância.

As colunas (5), (6) e (7) apresentam resultados para valores diferentes de gama, obtendo-se ótimos resultados a medida que é incrementado o valor de gama. As estimativas foram calculadas assumindo um tempo fixo dado por $t=1$. 


\section{CAPÍTULO 4}

\section{ESTIMAÇÃO BAYESIANA UTILIZANDO UMA FUNÇÃO DE PERDA LINEX}

\subsection{Introdução}

A estimação de funções de confiabilidade utilizando a perda assimétrica, vem sendo discutida com muito interesse nestes últimos anos. Segundo Canfield (1970), Basu e Ebrahimi (1988) e Hendrick Schäbe (1991), o uso da função de perda simétrica pode não ser satisfatória na obtenção dos estimadores.

$\mathrm{Na}$ prática, a função de perda real é frequentemente não simétrica. Superestimar a função de confiabilidade ou tempo médio de falha é normalmente muito mais grave que subestimar a função de confiabilidade ou tempo médio de falhas ou vice-versa. Por exemplo, na construção de uma represa, subestimar o nível da água é muito mais grave que sua superestimação.

Alguns autores, tais como Ferguson (1967), Zellner (1968) entre outros, utilizam funções de perdas assimétricas lineares. Varian (1975), por sua vez introduziu uma função de perda assimétrica conhecida como função de perda Linex. Esta função tem a forma exponencial no lado positivo e é quase linear no lado negativo. (ver Fig. 4.1).

O objetivo deste capítulo é desenvolver um método simples que nos permita obter estimativas Bayesianas para $\theta$ e $R(\theta)$, utilizando o modelo de distribuição exponencial, a função de perda Linex e um número de densidades a priori. Obteremos estimativas baseadas na função de perda quadrática e faremos uma comparação entre funções de risco, e de risco de Bayes para as funções de perdas Linex e quadrática. 


\subsection{Função de Perda Linex}

A função de perda Linex, introduzida por Varian (1975), é definida como:

$$
L(\Delta)=b e^{a \Delta}-c \Delta-b, \quad a, c \neq 0, \quad b>0,
$$

onde $\Delta=\left(\frac{\hat{\theta}}{\theta}-1\right)$ e $\hat{\theta}$ é o estimador de $\theta$.

Fazendo $a b=c$, a expressão (4.1) pode ser reformulada como :

$$
L(\Delta)=b\left[e^{a \Delta}-a \Delta-1\right], \quad a \neq 0, \quad b>0,
$$

onde $a$ é o parâmetro de forma e $b$ é o parâmetro de escala.

Para a função (4.2), quando $a=1$, a função é assimétrica com perda maior para a superestimação do que a subestimação (ver Fig. 4.1).

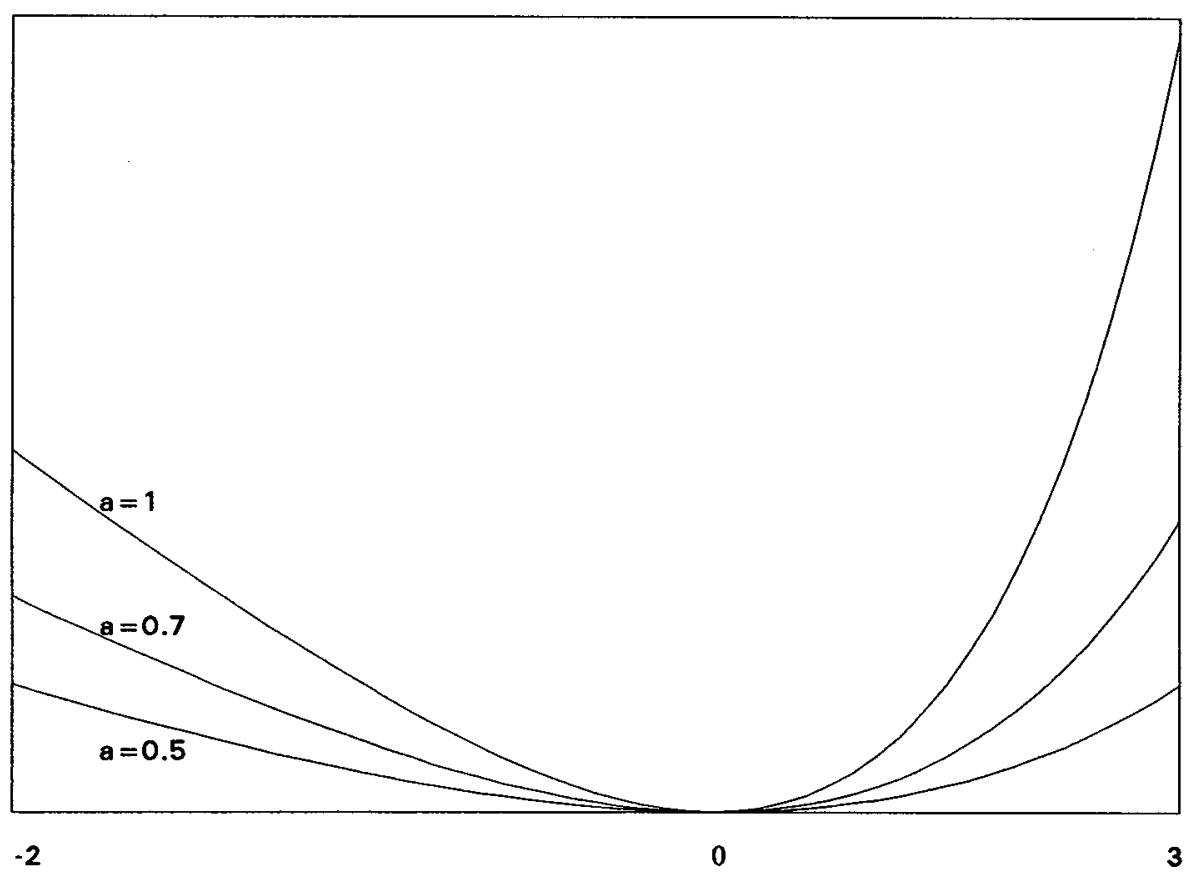

Fig 4.1 Função de Perda Linex 
Para valores pequenos de $|a|$ a função é quase simétrica e não distante da função quadrática, isto é, fazendo uma expansão de Taylor de $e^{a \Delta} \doteq 1+a \Delta+\frac{a^{2} \Delta^{2}}{2}$ e substituindo em (4.2), obtemos o seguinte resultado.

$$
L(\Delta)=\frac{a^{2} \Delta^{2}}{2} .
$$

Assim, para valores pequenos de $|a|$, as estimativas ótimas não são diferentes das estimativas baseadas na função de perda utilizada por El-Sayyad (1967).

\subsection{O Estimador de Bayes para $\theta$}

Nosso propósito, é obter o estimador de Bayes para o parâmetro $\theta$ que aparece na função de perda Linex (4.2). Para valores pequenos de $|a|$, a perda Linex comporta-se como a perda quadrática e neste caso o estimador de Bayes é a média da distribuição a posteriori.

Seja $p(\theta \mid D)$ a função de densidade a posteriori (f.d.p.) de $\theta$, onde $\mathrm{D}$ representa a amostra e a informação a priori e $E_{\theta}$ é a esperança da distribuição a posteriori.

O risco da distribuição a posteriori da função de perda Linex é definida como:

$$
R_{L}(\hat{\theta})=E_{\theta} L(\Delta)=\left[e^{-a} E_{\theta}\left(\exp \left(\frac{a \hat{\theta}}{\theta}\right)\right)-a E_{\theta}\left(\frac{\hat{\theta}}{\theta}-1\right)-1\right]
$$

Nosso interesse é procurar $\hat{\theta}$ que minimize (4.3), isto é, obtemos $\hat{\theta}$ que satisfaça a seguinte equação:

$$
\frac{\partial E_{\theta} L(\Delta)}{\partial \hat{\theta}}=e^{-a} a E_{\theta}\left(\frac{1}{\theta} \exp \left(\frac{\alpha \hat{\theta}}{\theta}\right)\right)-a E_{\theta}\left(\frac{1}{\theta}\right)=0 .
$$

Portanto, resolvendo a equação (4.4) em $\hat{\theta}$, o estimador de Bayes ( $\hat{\theta}_{B}$ ) é obtido da solução da seguinte equação:

$$
E_{\theta}\left(\frac{1}{\theta} \exp \left(\frac{a \hat{\theta}_{B}}{\theta}\right)\right)=e^{a} E_{\theta}\left(\frac{1}{\theta}\right),
$$


onde $E_{\theta}\left(\frac{1}{\theta} \exp \left(\frac{\alpha \hat{\theta}_{B}}{\theta}\right)\right)$ existe e é finito.

\subsection{Ostimador de Bayes para $\theta$ Utilizando uma Densidade a Priori não-informativa}

Os métodos Bayesianos, além de serem uma alternativa a soluções de problemas práticos, nos permite a incorporação de conhecimentos a priori na análise de dados, através de uma densidade a priori informativa.

Nesta seção, usaremos uma densidade a priori não-informativa para o parâmetro $\theta$, pois não existe informação proveniente dos dados. Neste caso, podemos utilizar uma priori quase uniforme, está densidade a priori é definida como:

$$
\pi_{1}(\theta)=\frac{1}{\theta^{d}}, \quad 0<\theta<\infty .
$$

Se $d=0$, obtemos a densidade a priori difusa, e se $d=1$ obtemos uma densidade a priori não-informativa.

Suponhamos que $n$ ítens da distribuição exponencial são colocados em teste e o experimento é realizado até que a r-ésima falha seja observada. Seja $t_{1}, \ldots, t_{r}$ uma amostra com censura tipo II, e denotando as primeiras $r$ observações ordenadas, neste caso obtemos a função de verossimilhança de $\theta$ que é dada pela seguinte expressão:

$$
l\left(t_{1}, \ldots, t_{r} \mid \theta\right)=\frac{n !}{(n-r) !} \frac{1}{\theta^{r}} \exp \left(-\frac{T_{r}}{\theta}\right)
$$

onde $T_{r}=\sum_{i=1}^{t} t_{i}+(n-r) t_{r}$. Considerando a densidade a priori não-informativa em (4.6) e a função de verossimilhança em (4.7) a densidade a posteriori para $\theta$ é dada por:

$$
p(\theta \mid D)=\frac{T_{r}^{(r+d-1)}}{\Gamma(r+d-1)}\left(\frac{1}{\theta}\right)^{r+d} \exp \left(-\frac{T_{r}}{\theta}\right), \quad(\theta>0, r+d>1) .
$$


Assim, desenvolvendo a expressão (4.5) e (4.8), obtemos o estimador de Bayes ótimo de $\theta$ para $L(\Delta)$, que é dado por:

$$
\hat{\theta}_{B}=\frac{Z}{a} T_{r},
$$

onde $Z=\left(1-e^{-\frac{a}{r+d}}\right)$

Também obtemos o estimador de Bayes para $\theta$ sob a perda quadrática, o qual é dado por,

$$
\hat{\theta}=\frac{1}{r+d-2} T_{r}, \quad(r+d>2) .
$$

Funções de risco são encontradas para $\hat{\theta}_{B}$ e $\hat{\theta}$ utilizando a função de perda Linex, e para efeitos de comparação também obtivemos as funções de risco para o estimador Bayesiano utilizando a função de perda quadrática definida como $L_{1}(\Delta)=(\hat{\theta}-\theta)^{2}$.

Para melhor ilustração, apresentaremos na tabela 4.1, um resumo da função de risco quando se usa a função de perda Linex e quadrática.

Tabela 4.1 Resultados da Função de Perda Linex e Quadrática.

\begin{tabular}{|c|c|}
\hline & FUNÇÃO DO RISCO \\
Estimador & A: Função de Perda Linex \\
\hline $\begin{array}{c}\text { Ótimo de Bayes } \\
\hat{\theta}_{B}\end{array}$ & $R_{L}\left(\hat{\theta}_{B}\right)=b\left[e^{-a}\left(\frac{1}{1-z}\right)^{r}-r z+a-1\right]$ \\
\hline $\begin{array}{c}\text { Média a Posteriori } \\
\hat{\theta}=\frac{1}{r+d-2} T_{r}\end{array}$ & $R_{L}(\hat{\theta})=b\left[e^{-a}\left(\frac{r+d-2}{r+d-a-2}\right)^{r}-\frac{r a}{r+d-2}+a-1\right],(\mathrm{r}+\mathrm{d}>\mathrm{a}+2)$ \\
\hline $\begin{array}{c}\text { Ótimo de Bayes } \\
\hat{\hat{\theta}_{B}}\end{array}$ & $R_{Q}\left(\hat{\theta}_{B}\right)=\frac{\hat{\theta}^{2}}{a^{2}}\left(z^{2} r(r+1)+a^{2}-2 a z r\right)$ \\
\hline$\hat{\theta}=\frac{1}{r+d-2} T_{r}$ & $R_{Q}(\theta)=\hat{\theta}^{2} \frac{\left(r+(d-2)^{2}\right)}{(r+d-2)^{2}},(r+d>2)$ \\
\hline
\end{tabular}




\subsubsection{Exemplo ( Martz e Waller, 1982)}

Um novo tipo de fechadura de válvula para portão é submetida a testes de vida acelerado para estimar o tempo médio de falha. Foram colocadas 20 válvulas sob teste, até que as 5 primeiras falhas ocorressem, o tempo total observado em teste para as cinco falhas é de $T_{5}=160000 h$. Segundo (Martz e Waller), assumimos que $d=a=1$. Os resultados do estimador de Bayes sob $L(\Delta)$ e a perda quadrática são apresentados na tabela 4.2.

Tabela 4.2 Resultados dos Estimadores Utilizando uma Densidade a Priori nãoInformativa

\begin{tabular}{|c|c|c|}
\hline Estimador & $\begin{array}{c}\text { Função do Risco } \\
\text { A:Função de Perda Linex }\end{array}$ & $\begin{array}{c}\text { Função do Risco } \\
\text { B:Funçãode Perda Quadrática }\end{array}$ \\
\hline$\hat{\theta}_{B}=24562.9$ & $\frac{R_{L}\left(\hat{\theta}_{B}\right)}{b}=0.08$ & $\frac{R_{Q}\left(\hat{\theta}_{B}\right)}{b}=0.172$ \\
\hline$\hat{\theta}=40000 h$ & $\frac{R_{L}(\hat{\theta})}{b}=0.300$ & $\frac{R_{Q}\left(\hat{\theta}_{B}\right)}{b}=0.375$ \\
\hline
\end{tabular}

Como podemos observar o risco associado sob a função de perda Linex com $\hat{\theta}$ é de 0.22 maior que o risco associado com $\hat{\theta}_{B}$. Sob a perda quadrática o risco associado com $\hat{\theta}$ é $20 \%$ maior que o risco associado com $\hat{\theta}_{B}$.

\subsection{O Estimador de Bayes para $\theta$ Utilizando uma Densidade a Priori Gama Invertida}

Segundo, Raiffa e Schlaifer (1961), Bhattacharyya (1967) e outros afirmam que a densidade a priori Gama Invertida é amplamente utilizada em confiabilidade. A principal razão de utilizar está priori, é que os resultados obtidos são matematicamente tratáveis devido ao fato que a densidade Gama Invertida é uma a priori conjugada para $\theta$. A densidade Gama Invertida com parâmetros $\alpha$ e $\beta>0$, é definida como:para $\theta$.

$$
\pi_{2}(\theta)=\frac{\beta^{\alpha}}{\Gamma(\alpha)}\left(\frac{1}{\theta}\right)^{\alpha+1} \exp \left(-\frac{\beta}{\theta}\right), \quad \theta>0
$$


Utilizando a função de verossimilhança (4.7) e uma densidade a priori Gama Invertida (4.11), obtemos a distribuição a posteriori para $\theta$ que é dada pela seguinte expressão:

$$
p(\theta \mid D)=\frac{\left(T_{r}+\beta\right)^{r+\alpha}}{\Gamma(r+\alpha)}\left(\frac{1}{r}\right)^{r+\alpha+1} \exp \left(-\frac{T_{r}+\beta}{\theta}\right), \quad 0<\theta<\infty
$$

A distribuição (4.12) é uma Gama Invertida com parâmetros $\left(r+\alpha, \beta+T_{r}\right)$.

Assim, utilizando (4.11) e (4.12) e fazendo os respectivos cálculos, obtemos o estimador de Bayes ótimo $\left(\hat{\theta}_{B}\right)$ sob a a função de perda Linex $L(\Delta)$,

$$
\hat{\theta}_{B}=\frac{Z_{1}}{a}\left(T_{r}+\beta\right)
$$

onde $Z_{1}=1-\exp \left(-\frac{a}{r+\alpha-1}\right)$

Também, obtivemos o estimador de Bayes para $\theta$ sob a perda quadrática:

$$
\hat{\theta}=\frac{T_{r}+\beta}{r+\alpha-1} . \quad(r+\alpha>1) .
$$

A seguir, apresentaremos os resultados da função de risco e função de risco de Bayes, para $\hat{\theta}_{B}$ e $\hat{\theta}$, utilizando a função de perda Linex e a função de perda quadrática.

$\mathrm{Na}$ tabela (4.3), apresentamos um resumo dos principais estimadores que foram obtidos. 


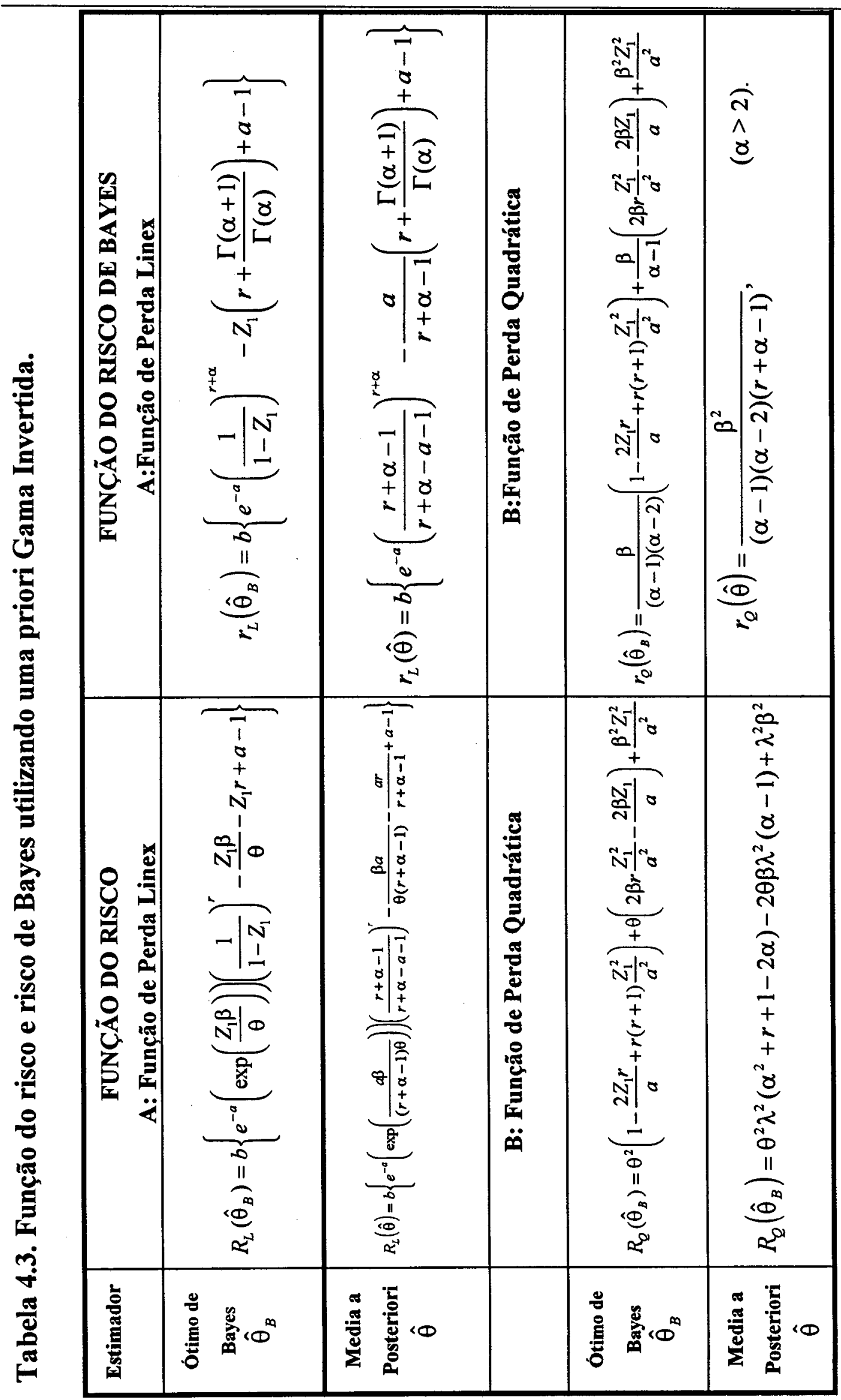




\subsubsection{Exemplo (Considerando o exemplo 4.4.1)}

Neste exemplo, utilizamos uma densidade a priori Gama Invertida com $\alpha=8.5$ e $\beta=2.86 \times 10^{5}$ a qual foi sugerida por Martz e Waller (1982). Os resultados do estimador de Bayes para $\hat{\theta}_{\mathrm{B}}$ e $\theta$, segundo a função de perda Linex e a perda quadrática, são apresentados na tabela (4.4).

Tabela 4.4 Resultados dos Estimadores Utilizando uma Densidade a Priori Gama Invertida.

\begin{tabular}{|c|c|c|}
\hline Estimador & $\begin{array}{c}\text { FUNÇÃo DO RISCO } \\
\text { A: Função de Perda Linex }\end{array}$ & $\begin{array}{c}\text { FUNÇÃO DO RISCO DE BAYES } \\
\text { A: Função de Perda Linex }\end{array}$ \\
\hline$\hat{\theta}_{B}=29721.95$ & $\frac{R_{L}\left(\hat{\theta}_{B}\right)}{b}=0.053$ & $\frac{r_{L}\left(\hat{\theta}_{B}\right)}{b}=0.0391$ \\
\hline$\hat{\theta}=35680$ & $\frac{R_{L}(\hat{\theta})}{b}=0.28$ & $\frac{r_{L}(\hat{\theta})}{b}=0.06$ \\
\hline & B: Função de Perda Quadrática & B: Função de Perda Quadrática \\
\hline$\hat{\theta}_{B}=29721.95$ & $R_{Q}\left(\hat{\theta}_{B}\right)=0.0926$ & $r_{Q}\left(\hat{\theta}_{B}\right)=89 \times 10^{7}$ \\
\hline$\hat{\theta}=35680$ & $R_{Q}(\hat{\theta})=0.4$ & $r_{Q}(\hat{\theta})=13.4 \times 10^{7}$ \\
\hline
\end{tabular}

$\mathrm{Na}$ tabela 4.4, observamos que os resultados são ótimos quando utilizamos o estimador de Bayes na função de perda Linex.

Quando fazemos $\alpha=a=\theta=1, \beta=2$ e $r=5$, na função de risco, os estimadores segundo a perda quadrática passam a ser melhores que os estimadores segundo a perda Linex.

Também encontramos que a razão do risco mínimo de Bayes associado com $\hat{\theta}_{B}$ para $\hat{\theta}$ é 0.65 sob a função de perda linex e a razão sob a perda quadrática é 6.6. Portanto na função de perda linex o risco de Bayes para $\hat{\theta}$ é $53 \%$ maior que o risco para $\hat{\theta}_{B}$, e na perda quadrática o risco de Bayes para $\hat{\theta}$ é de $15 \%$ maior que o risco para $\hat{\theta}_{B}$. 


\subsection{O Estimador de Bayes para $\theta$ Utilizando uma Densidade a Priori Uniforme}

Freqüentemente ocorre que em experimentos sobre testes de vida, o valor provável do parâmetro $\theta$ encontra-se em um intervalo finito $[\alpha, \beta]$, porém não existe conhecimento sobre algum subconjunto de valores neste intervalo. Em tais circunstâncias, uma densidade uniforme de $\theta$ sobre o intervalo acima mencionado, pode servir como uma razoável aproximação para representar a densidade a priori da opinião do experimentador. A densidade a priori Uniforme é definida como:

$$
\pi_{3}(\theta)=\frac{1}{\beta-\alpha} . \quad 0<\alpha \leq \theta<\beta
$$

Utilizando a densidade a priori Uniforme (4.14) e a função de verossimilhança (4.7), encontramos uma distribuição a posteriori para $\theta$ definida como:

$p(\theta \mid D)=\left(\exp \left\{-\frac{T_{r}}{\theta}\right\}\right)\left(T_{r}\right)^{r-1} \frac{1}{\theta^{r}}\left[\Gamma\left(r-1, \frac{T_{r}}{\alpha}\right)-\Gamma\left(r-1, \frac{T_{r}}{\beta}\right)\right]^{-1}, \quad 0<\alpha \leq \theta \leq \beta$

onde $\Gamma(n, x)=\int_{0}^{x}(\exp (-t)) t^{n-1} d t$ é a função da Gama Incompleta com $(n>0)$.

O estimador de Bayes de $\hat{\theta}_{B}$ relativo sob a função de perda Linex é obtido utilizando a expressão (4.5) e (4.15). Portando $\hat{\theta}_{B}$ é a solução da seguinte equação:

$$
e^{a} \frac{\Gamma\left(r, \frac{T_{r}}{\alpha}\right)-\Gamma\left(r, \frac{T_{r}}{\beta}\right)}{\Gamma\left(r, \frac{T_{r}-a \hat{\theta}_{b}}{\alpha}\right)-\Gamma\left(r, \frac{T_{r}-a \hat{\theta}_{B}}{\beta}\right)}=\left(\frac{T_{r}}{T_{r}-a \hat{\theta}_{B}}\right)^{r}
$$

Os métodos Bayesianos estão sujeitos a algumas limitações relacionadas a obtenção explícita dos estimadores (ver equação 4.16). Para resolvermos este problema surge a possibilidade de utilizar métodos iterativos, neste caso utilizamos o método iterativo "Newton-Raphson"," o qual nos permite encontrar soluções ótimas para os estimadores.

* Ver apêndice $B$ 
Finalizando, obtemos o estimador de Bayes sob a perda quadrática, que é dado por,

$$
\hat{\theta}=\left[\frac{\Gamma\left(r-2, \frac{T_{r}}{\alpha}\right)-\Gamma\left(r-2, \frac{T_{r}}{\beta}\right)}{\Gamma\left(r-1, \frac{T_{r}}{\alpha}\right)-\Gamma\left(r-1 . \frac{T_{r}}{\beta}\right)}\right] T_{r} .
$$

Observação: As funções de risco $R_{L}\left(\hat{\theta}_{B}\right), R_{L}(\hat{\theta}), R_{Q}\left(\hat{\theta}_{B}\right), R_{Q}(\hat{\theta})$ e os correspondentes risco de Bayes não podem ser obtidos de forma explícita.

\subsubsection{Exemplo (Continuação do exemplo 4.4.1)}

Neste caso, consideramos uma distribuição a priori uniforme para $\theta$ com $\alpha=2 \times 10^{4}$ e $\beta=7 \times 10^{4}$ como foi sugerida por Martz e Waller (1982). Utilizando as equações (4.16) e (4.17) encontramos os resultados para o estimador de Bayes:

$$
\hat{\theta}_{B} \doteq 26358.38 h \quad \hat{\theta}=41100 h .
$$

Como podemos observar, o estimador de Bayes do tempo médio de falha sob a perda Linex, é menor que o estimador de Bayes do tempo médio de falha sob a perda quadrática.

\subsection{O Estimador de Bayes para $R(t)$}

\subsubsection{O Estimador de Bayes Utilizando uma Densidade a Priori não-informativa}

Segundo Edward Pugh (1962), Paul Chiou (1993) e outros, a distribuição exponencial tem grande aplicação em modelos de sobrevivência, pois representa um modelo apropriado para descrever a confiabilidade e o sistema de falhas de componentes, quando estes não apresentam variações em suas taxas de falha. A função de densidade para um modelo exponencial é definida como: 


$$
f(t \mid \theta)=\frac{1}{\theta} \exp \left(-\frac{t}{\theta}\right), \quad t>0, \theta>0
$$

onde o parâmetro $\theta$ é o tempo médio de falhas. Nesta seção desenvolvemos algumas técnicas para estimar a confiabilidade (função de sobrevivência) dada por $R=R(t)=\exp \left(-\frac{t}{\theta}\right)$

Para situações quando o experimento não tem uma informação a priori acerca de $R$, surge a possibilidade de utilizar uma densidade a priori não-informativa, conhecida também como a priori de Jeffreys (1967), (ver Box \& Tiao 1973) e esta densidade é definida como:

$$
\pi_{1}(R)=\frac{1}{R(-\log R)} . \quad 0<R<1 .
$$

Suponhamos, que $t_{1}, \ldots, t_{r}$ denotando as $r$ primeiras observações de uma amostra com censurada tipo II, portanto a função de verossimilhança condicional sobre $R$ é dada por:

$$
l\left(t_{1}, \ldots, t_{r} \mid R\right)=\frac{n !}{(n-r) !}\left(-\frac{1}{t} \log R\right)^{r}(R)^{\frac{T_{r}}{t}}
$$

onde $T_{r}=\sum_{i=1}^{r} t_{i}+(n-r) t_{r}$. Utilizando a densidade a priori (4.19) e a função de verossimilhança (4.20), obtemos a distribuição a posteriori dada por:

$$
p(R \mid D)=\frac{(-\log R)^{r-1}(R)^{w-1} w^{r}}{\Gamma(r)}
$$

onde $w=\frac{T_{r}}{t}$.

Zellner (1986), utilizou a função de perda assimétrica definida como:

$$
L\left(\Delta_{1}\right)=b\left[e^{a(\hat{R}-R)}-a(\hat{R}-R)-1\right],
$$

onde $\Delta_{1}=\hat{R}-R$. 
A função de perda Linex é assimétrica quando $a=1$. Também, quando $a<0, L\left(\Delta_{1}\right)$ aumenta exponencialmente com $\Delta_{1}=\hat{R}-R<0$, e é quase linear com $\Delta_{1}=\hat{R}-R>0$. Para valores pequenos de $|a|, L\left(\Delta_{1}\right)$ não é distante da função de perda quadrática $L(\hat{R}, R)=(\hat{R}-R)^{2}$.

O estimador de Bayes para a confiabilidade $\mathbf{R}$ é obtido aplicando o valor esperado na função de perda Linex (4.22). Assim obtemos:

$$
E_{R} L\left(\Delta_{1}\right)=b\left[e^{a \hat{R}} E_{R}\left(e^{-a R}\right)-a \hat{R}+a E_{R}(R)-1\right] .
$$

Logo, derivando em relação a $\hat{R}$ e fazendo os respectivos cálculos obtemos:

$$
\frac{\partial E_{R} L\left(\Delta_{1}\right)}{\partial \hat{R}}=b\left[a e^{a \hat{R}} E_{R}\left(e^{-a R}\right)-a\right]=0
$$

onde,

$$
\hat{R}=-\frac{1}{a} \log \left[E_{R}\left(e^{-a R}\right)\right]
$$

Desenvolvendo:

$$
\begin{aligned}
& E_{R}\left(e^{-a R}\right)=E_{R}\left[\sum_{j=0}^{\infty} \frac{(-a R)^{j}}{j !}\right]=E_{R}\left[\sum_{j=0}^{\infty} \frac{(-a)^{j}}{j !} R^{j}\right], \\
& E_{R}\left(e^{-a R}\right)=\sum_{j=0}^{\infty} \frac{(-a)^{j}}{j !} E_{R}\left(R^{j}\right) .
\end{aligned}
$$

Os valores acima encontrados são substituídos em (4.23) e obtemos:

$$
\hat{R}=-\frac{1}{a} \log \left\{\sum_{j=0}^{\infty} \frac{(-a)^{j}}{j} E_{R}(R)^{J}\right\},
$$

onde,

$$
E_{R}\left(R^{j}\right)=\int_{0}^{1} R^{j} p(R \mid D) d R=\int_{0}^{1} \frac{w^{r}}{\Gamma(r)}(-\log R)^{r-1} R^{w-1+j} d R .
$$


Desenvolvendo (4.25) e substituindo os resultados em (4.24), obtemos o estimador de Bayes para a confiabilidade que é dado por:

$$
\hat{R}_{B}=-\frac{1}{a} \log \left\{\sum_{j=0}^{\infty} \frac{(-a)^{j}}{j !}\left(1+\frac{j}{w}\right)^{-r}\right\}
$$

O estimador ótimo utilizando a perda quadrática é dado pela seguinte expressão:

$$
\hat{R}=\left(1+\frac{1}{w}\right)^{-r}
$$

\section{Observação :}

Como pode ser observado as funções de risco $R_{L}\left(\hat{R}_{B}\right), R_{L}(\hat{R}), R_{Q}\left(\hat{R}_{B}\right), R_{Q}(\hat{R})$ e os correspondentes riscos de Bayes não podem ser obtidos de forma explícita.

\subsubsection{O Estimador de Bayes Utilizando uma Densidade a Priori Beta $(\alpha, \beta)$}

Uma densidade amplamente utilizada em confiabilidade é a densidade a priori Beta com parâmetros $(\alpha, \beta)$ e é definida como:

$$
\pi_{2}(R)=\frac{1}{\mathrm{~B}(\alpha, \beta)}(R)^{\alpha-1}(1-R)^{\beta-1}, \quad 0<R \leq 1 .
$$

Considerando uma densidade a priori (4.28) e uma função de verossimilhança (4.20), obtemos a distribuição a posteriori para a confiabilidade $R$ dada por:

$$
p(R \mid D)=\frac{(-\log R)^{r}(R)^{\alpha+w-1}(1-R)^{\beta-1}}{\Gamma(r+1) \sum_{K=0}^{\beta-1}(-1)^{k}\left(\begin{array}{c}
\beta-1 \\
K
\end{array}\right) \frac{1}{(\alpha+w+k)^{r+1}}} . \quad 0<R \leq 1
$$

$\mathrm{O}$ estimador de Bayes para a confiabilidade $\mathrm{R}$ é obtido aplicando o valor esperado na função de perda Linex (4.22). Assim obtemos:

$$
E_{R} L\left(\Delta_{1}\right)=b\left[e^{a \hat{R}} E_{R}\left(e^{-a R}\right)-a \hat{R}+a E_{R}(R)-1\right]
$$


Logo, derivando em relação a $\hat{R}$ e fazendo os respectivos cálculos obtemos:

$$
\hat{R}=-\frac{1}{a} \log \left[E_{R}\left(e^{-a R}\right)\right]
$$

Sabemos que :

$$
\begin{aligned}
& E_{R}\left(e^{-a R}\right)=E_{R}\left[\sum_{j=0}^{\infty} \frac{(-a R)^{j}}{j !}\right]=E_{R}\left[\sum_{j=0}^{\infty} \frac{(-a)^{j}}{j !} R^{j}\right] \\
& E_{R}\left(e^{-a R}\right)=\sum_{j=0}^{\infty} \frac{(-a)^{j}}{j !} E_{R}\left(R^{j}\right),
\end{aligned}
$$

onde, $\quad E_{R}\left(R^{j}\right)=\int_{0}^{1} \frac{(-\log R)^{r}(R)^{\alpha+w-1}(1-R)^{\beta-1}}{\Gamma(r+1) \sum_{k=0}^{\beta-1}(-1)^{k}\left(\begin{array}{c}\beta-1 \\ k\end{array}\right) \frac{1}{(\alpha+w+k)^{r+1}}} d R$.

O estimador de Bayes é obtido em relação a perda Linex depois de utilizar (4.31) em (4.30), e fazendo os respectivos cálculos obtemos:

$$
\hat{R}_{B}=-\frac{1}{a} \log \left\{\frac{\sum_{j=0}^{\infty} \sum_{k=0}^{\beta-1}(-1)^{j}(-1)^{k}\left(\begin{array}{c}
\beta-1 \\
k
\end{array}\right) \frac{1}{(j+k+w+\alpha)^{r+1}}}{\sum_{k=0}^{\beta-1}(-1)^{k}\left(\begin{array}{c}
\beta-1 \\
k
\end{array}\right) \frac{1}{(k+w+\alpha)^{r+\alpha}}}\right\}
$$

O estimador de Bayes da confiabilidade sob a perda quadrática é dado por,

$$
\hat{R}=\frac{\sum_{k=0}^{\beta-1}\left(\begin{array}{c}
\beta-1 \\
k
\end{array}\right)(-1)^{k} \frac{1}{(\alpha+w+1+k)^{r+1}}}{\sum_{k=0}^{\beta-1}(-1)^{k}\left(\begin{array}{c}
\beta-1 \\
k
\end{array}\right) \frac{1}{(k+w+\alpha)}}
$$

Observação: As funções de risco $R_{L}\left(\hat{R}_{B}\right), R_{L}(\hat{R}), R_{Q}\left(\hat{R}_{B}\right), R_{Q}(\hat{R})$ e os correspondentes ricos de Bayes não podem ser obtidos de forma explícita. 
Também, podemos concluir que o estimador de Bayes para confiabilidade obtido neste capítulo apresenta uma vantagem em termos de cálculos, em relação ao estimador obtido por Canfield (1970), dado no capítulo 3, pois este estimador apresenta muitas dificuldades computacionais.

\subsubsection{Exemplo (Continuação do exemplo 4.4.1)}

Neste exemplo, estimaremos $R$ com $t=4000 h$. Quando se utiliza uma densidade a priori não-informativa, os resultados do estimador de Bayes em relação a perda Linex $L\left(\Delta_{1}\right)$ são:

$$
\hat{R}_{B} \doteq 0.788, \quad \hat{R}=0.8839 .
$$

Quando utilizamos uma densidade a priori Beta $\operatorname{com} \alpha=27$ e $\beta=3$, foram obtidos o estimador de Bayes segundo a perda Linex e a função de perda quadrática, e os resultados são:

$$
\hat{R}_{B} \doteq 0.793 \quad \text { e } \quad \hat{R}=0.8897 \text {. }
$$

Em ambos resultados, o estimador de Bayes da função de confiabilidade segundo a perda linex é menor em relação ao estimador de Bayes segundo a perda quadrática.

Em muitas situações as funções de perdas assimétricas envolvem estimativas para a confiabilidade e para o tempo médio de falhas. Estas estimativas, obtidas neste capítulo, são mais apropriadas que as estimativas obtidas segundo a perda quadrática, como foi analisado no decorrer deste capítulo são completamente diferentes. 


\section{CAPÍtulo 5}

\section{FUNÇÃO DE PERDA BALANCEADA PONDERADA COM DADOS CENSURADOS}

\subsection{Introdução}

Neste capítulo, desenvolveremos um procedimento que estabelece uma relação entre a estatística clássica e a Bayesiana, que pode simplificar de uma forma objetiva as interpretações e conclusões de problemas reais.

Geralmente, em problemas estatísticos, é possível utilizar as funções de perdas para medir o quanto perdemos ao fazer inferências a respeito do parâmetro $\theta$. Desenvolveremos um método de estimação baseado na Função de Perda Balanceada Ponderada (FPBP), a qual reflete simultaneamente o ajuste do modelo e a precisão do estimador. A Função de Perda Balanceada (FPB) foi introduzida por Zellner (1991), para estimar a média de uma população normal, e depois foi generalizada por Rodrigues \& Zellner (1992) para estimar a média de um modelo exponencial sem censura. Nosso interesse é estender a função de perda de Rodrigues \& Zellner (1992), incluindo censura do tipo II e uma função de perda não simétrica para caracterizar a precisão do estimador.

Na seção 2, supomos uma priori não-informativa e obtivemos o estimador ótimo da Função de Perda Balanceada Ponderada (FPBP). A seguir, concluímos com uma comparação, em termos da eficiência relativa do estimador clássico com o estimador ótimo dado pela Função de Perda Balanceada Ponderada (FPBP). Sugerimos, também, um 
estimador para a função de confiabilidade baseado na Função de Perda Balanceada (FPBP) proposta neste capitulo.

\subsection{Obtenção do Estimador Ótimo Utilizando a Função de Perda Balanceada Ponderada (FPBP)}

A inferência clássica em geral utiliza o método dos mínimos quadrados para medir o ajuste do modelo ou a perda quadrática para medir a precisão do estimador (Zellner (1991)). Neste capítulo, propomos uma função de perda assimétrica, chamada função de perda Linex, a qual será utilizada para medir a precisão do estimador. Esta função de perda foi definida por Varian (1975) e utilizada por Zellner (1986).

Suponhamos que temos uma amostra com distribuição exponencial, onde $X_{i} \sim \varepsilon(\theta)$ $i=1, \ldots, n$, isto é,

$$
f(x \mid \theta)=\frac{1}{\theta} \mathrm{e}^{-x / \theta}, x>0, \theta>0
$$

e que os dados estejam sujeitos a uma censura de tipo II (ver Lawless, 1983), onde $r \leq n$ é o número de falhas admitido na amostra. A Função de Perda Balanceada Ponderada (FPBP) para $\theta$, denotada por $L_{B}(\hat{\theta}, \theta)$, onde $\hat{\theta}$ é o estimador de $\theta$, é definida como:

$L_{B}(\hat{\theta}, \theta)=w\left[\frac{\sum_{i=1}^{n}\left(X_{i}-\hat{\theta}\right)^{2}}{r \hat{\theta}^{2}}\right]+(1-w)\left[b\left(e^{\Delta \Delta}-a \Delta-1\right)\right], \quad a \neq 0, b>0$

onde, $\Delta=\left(\frac{\hat{\theta}}{\theta}-1\right)$ e $0 \leq w \leq 1$.

O primeiro termo em (5.2) representa o ajuste do modelo censurado, e o segundo termo a precisão da estimação segundo a perda Linex. Logo, utilizando a função de distribuição a posteriori para $\theta, p(\theta \mid D)$, obtemos o risco a posteriori de (5.2) que é dado por : 
$E_{\theta} L_{B}(\hat{\theta}, \theta)=w\left[\frac{\bar{s}^{2}}{\hat{\theta}^{2}}+\frac{n}{r}\left(\frac{\hat{\theta}-\tilde{x}}{\hat{\theta}}\right)^{2}\right]+(1-w)\left[E_{\theta}\left(e^{a\left(\frac{\hat{\theta}}{\theta}-1\right)}\right)-a E_{\theta}\left(\frac{\hat{\theta}}{\theta}-1\right)-1\right]$

onde, $\bar{s}^{2}=\frac{\sum_{i=1}^{n}\left(X_{i}-\tilde{x}\right)^{2}}{r}$ é a variância dos dados censurados e $\tilde{x}=\frac{T r}{r}$ é o estimador de maxima verossimilhança de $\theta$ (média amostral censurada), sendo $T r$ : tempo total observado e r: número das observações ordenadas.

Para $w=1$, temos o ajuste de mínimos quadrados e para $w=0$ a função de perda Linex proposta por Zellner, (1986).

Fazendo algumas simplificações algébricas em (5.3) e derivando com respeito a $\hat{\theta}$ obtemos a seguinte expressão:

$$
\frac{\partial E_{\theta} L_{B}(\hat{\theta}, \theta)}{\partial \hat{\theta}}=w\left[\frac{-2 \vec{s}^{2}}{\hat{\theta}^{3}}+\frac{2 n}{r}\left[\frac{(\hat{\theta}-\tilde{x})}{\hat{\theta}^{2}}-\frac{(\hat{\theta}-\tilde{x})^{2}}{\hat{\theta}^{3}}\right]\right]+(1-w)\left[e^{-a} a E_{\theta}\left(\frac{e^{\frac{\partial \hat{\theta}}{\theta}}}{\theta}\right)-a E_{\theta}\left(\frac{1}{\theta}\right)\right]
$$

Igualando a zero a expressão (5.4), isto é, $\frac{\partial E_{\theta} L_{B}(\hat{\theta}, \theta)}{\partial \hat{\theta}}=0$, podemos obter o estimador ótimo balanceado para $\theta$, para $0 \leq w \leq 1$, que representamos por $\hat{\theta}_{*}$.

Quando temos $w=1$ em (5.4), o estimador ótimo balanceado pontual coincide com o estimador clássico definido por,

$$
\hat{\theta}_{c}=\frac{\bar{s}^{2}+\frac{n}{r} \tilde{x}^{2}}{\frac{n}{r} \tilde{x}} .
$$

Analogamente, quando $w=0 \mathrm{em}$ (5.4), obtemos o estimador pontual Bayesiano dado por:

$$
\hat{\theta}_{B}=\frac{\left(1-e^{\left(-\frac{a}{r+d}\right)}\right) \operatorname{Tr}}{a}
$$


onde a densidade a priori é não informativa e é dada por $p(\theta) \propto \frac{1}{\theta}$.

Em geral, os métodos bayesianos estão sujeitos a limitações para a obtenção de soluções analíticas explícitas.

Neste caso, para obter o estimador ótimo balanceado, baseado na Função de Perda Balanceada Ponderada (FPBP), utilizamos o algoritmo "Newton-Raphson" (ver, Kalbfleisch, 1985) que é dado por:

$$
\theta_{(i)}=\theta_{(i-1)}-\frac{f\left(\theta_{(i-1)}\right)}{f^{\prime}\left(\theta_{(i-1)}\right)},
$$

onde,

$$
\begin{aligned}
& f(\theta)=w\left[\frac{-2\left(\bar{s}^{2}+\frac{n}{r} \tilde{x}^{2}\right)}{\hat{\theta}^{3}}+\frac{2 n \tilde{x}}{\hat{\theta}^{2}}\right]+(1-w)\left[\frac{e^{-a} a \operatorname{Tr}^{(r+d-1)}(r+d-1)}{(\operatorname{Tr}-a \hat{\theta})}-\frac{a(r+d-1)}{T r}\right] \\
& f^{\prime}(\theta)=w\left[\frac{6\left(\bar{s}^{2}+\frac{n}{r} \tilde{x}^{2}\right)}{\hat{\theta}^{4}}-\frac{4 n \tilde{x}}{r \hat{\theta}^{3}}\right]+(1-w)\left[\frac{e^{-a} a^{2} \operatorname{Tr}^{(r+d-1)}(r+d-1)(r+d)}{(\operatorname{Tr}-a \hat{\theta})^{(r+d-1)}}\right] .
\end{aligned}
$$

É importante salientar que este algoritmo está baseado na densidade a priori nãoinformativa dada por $p(\theta) \propto \frac{1}{\theta}$.

Para ilustrar o comportamento do procedimento balanceado, geramos uma amostra * censurada do modelo exponencial com $\theta=3, n=20, r=16$ e $a=d=1$. Obtendo $\tilde{x}=5.91837, \operatorname{Tr}=94.694$ e $\overline{\mathrm{s}}^{2}=13.1597$.

Os resultados obtidos para o estimador ótimo balanceado e para diferentes valores de $w$ serão apresentados na Tabela 5.1. Para verificação do algoritmo, utilizamos os dados do livro (Lawless, 1983, pág.: 103), e obtivemos os resultados esperados.

\footnotetext{
${ }^{*}$ Ver apêndice $\mathrm{C}$
} 


\subsection{Comparação do Estimador Ótimo Balanceado com o Estimador Clássico}

Para comparar o estimador ótimo balanceado com o estimador clássico, vamos utilizar a perda média a posteriori que é dada por,

$E_{\theta} L_{B}(\hat{\theta}, \theta)=w\left[\frac{\bar{s}^{2}}{\hat{\theta}^{2}}+\frac{n}{r}\left(\frac{\hat{\theta}-\tilde{x}}{\hat{\theta}}\right)^{2}\right]+e^{-a} E_{\theta}\left(e^{\frac{a \hat{\theta}}{\theta}}\right)-a E_{\theta}\left(\frac{\hat{\theta}}{\theta}\right)-w e^{-a} E_{\theta}\left(e^{\frac{\hat{\theta}}{\theta}}\right)+w a E_{\theta}\left(\frac{\hat{\theta}}{\theta}\right)$.

Supondo uma priori não-informativa podemos demostrar que:

$$
e^{-a} E_{\theta}\left(e^{\frac{\hat{\theta}}{\theta}}\right)=\frac{e^{-a} \operatorname{Tr}^{(r+d-1)}}{(\operatorname{Tr}-a \hat{\theta})^{(r+d-s)}}
$$

e que

$$
a E_{\theta}\left(\frac{\hat{\theta}}{\theta}\right)=\frac{\hat{\theta}(r+d-1)}{T r}
$$

Substituindo as expressões (5.8) e (5.9) em (5.7), temos que a perda média a posteriori (5.7) é dada por:

$E_{\theta} L_{B}(\hat{\theta}, \theta)=w\left[\frac{\bar{s}^{2}}{\hat{\theta}^{2}}+\frac{n}{r}\left(\frac{\hat{\theta}-\tilde{x}}{\hat{\theta}}\right)^{2}\right]+(1-w)\left[\frac{e^{-a} \operatorname{Tr}^{(r+d-1)}}{(\operatorname{Tr}-a \hat{\theta})^{(r+d-s)}}-\frac{\hat{d}(r+d-1)}{\operatorname{Tr}}\right]$

Nosso objetivo é comparar o estimador ótimo balanceado $\hat{\theta}_{*}$, baseado nos dados que foram simulados anteriormente, com o estimador clássico. Substituindo o estimador clássico (5.5) em (5.10) obtemos o seguinte resultado: 


$$
\begin{aligned}
E_{\theta} L_{B}\left(\hat{\theta}_{c}, \theta\right)= & w\left[\frac{n^{2} \tilde{x}^{2} \bar{s}^{2}+n r\left(\bar{s}^{2}\right)^{2}}{\left(r \bar{s}^{2}+n \tilde{x}^{2}\right)^{2}}\right]+ \\
& +(1-w)\left[\frac{e^{-a} \operatorname{Tr}^{(r+d-1)}(n \tilde{x})^{(r+d-1)}}{\left(n \tilde{x} T r-a\left(r \bar{s}^{2}+n \tilde{x}^{2}\right)\right)^{(r+d-1)}}-\frac{a\left(r \bar{s}^{2}+n \tilde{x}^{2}\right)(r+d-1)}{T r}\right] .
\end{aligned}
$$

Analogamente, substituindo o estimador ótimo balanceado $\hat{\theta}_{*}$, em (5.10) temos que:

$$
E_{\theta} L_{B}\left(\hat{\theta}_{*}, \theta\right)=w\left[\frac{\bar{s}^{2}}{\hat{\theta}_{*}^{2}}+\frac{n}{r}\left(\frac{\hat{\theta}_{*}-\tilde{x}}{\hat{\theta}_{*}}\right)^{2}\right]+(1-w)\left[\frac{e^{-a} \operatorname{Tr}^{(r+d-1)}}{\left(T r-d \hat{\theta}_{*}\right)^{(r+d-s)}}-\frac{d \hat{\theta}_{*}(r+d-1)}{T r}\right]
$$

Utilizando as expressões (5.11) e (5.12) é possível comparar os estimadores clássico e Bayesiano através do fator $\Delta$ definido por:

$$
\begin{aligned}
& \Delta_{c}=E_{\theta} L_{B}\left(\hat{\theta}_{c}, \theta\right)-E_{\theta} L_{B}\left(\hat{\theta}_{*}, \theta\right), \\
& \Delta_{B}=E_{\theta} L_{B}\left(\hat{\theta}_{B}, \theta\right)-E_{\theta} L_{B}\left(\hat{\theta}_{*}, \theta\right),
\end{aligned}
$$

onde o fator $\Delta_{c}$ e $\Delta_{\mathrm{B}}$, medem o aumento da perda esperada a posteriori associada ao uso do estimador clássico ou Bayesiano, em lugar do estimador ótimo balanceado $\hat{\theta}_{*}$.

Além disso, para efeitos de comparação, segundo (Zellner, 1991), é mais ilustrativo comparar os estimadores em termos da eficiência relativa, $\left(E_{c}\right.$ e $\left.E_{B}\right)$, que são dados por:

$$
\begin{aligned}
& E_{c}=\frac{\Delta_{c}}{E_{\theta} L_{B}\left(\hat{\theta}_{*}, \theta\right)} \\
& E_{B}=\frac{\Delta_{B}}{E_{\theta} L_{B}\left(\hat{\theta}_{*}, \theta\right)}
\end{aligned}
$$

Resultados obtidos serão apresentados na tabela 5.1, quandos se faz uso de uma Função de Perda Balanceada Pondera, e na tabela 5.2 apresentamos os resultados quando se faz variar o parâmetro de forma $a$. 
Tabela 5.1. Resultados Obtidos para o Estimador Ótimo Baseados em $\theta=3, n=20, r=16, a=d=1$ e $t=40$.

\begin{tabular}{|c|c|c|c|c|}
\hline$w$ & $\hat{\theta}_{*}$ & $E_{c}=\frac{\Delta_{c}}{E_{\theta} L_{B}\left(\hat{\theta}_{*}, \theta\right)}$ & $E_{B}=\frac{\Delta_{B}}{E_{\theta} L_{B}\left(\hat{\theta}_{*}, \theta\right)}$ & $\hat{R}(t)$ \\
\hline 0 & 5.40957 & 3.42794338 & 0 & 0.0006147 \\
\hline 0.25 & 6.24674 & 0.45134874 & 0.196633942 & 0.0016560 \\
\hline 0.50 & 6.71429 & 0.04279133 & 0.200504102 & 0.0025865 \\
\hline 0.75 & 7.14638 & 0.02790211 & 0.458732374 & 0.0037081 \\
\hline 1 & 7.69721 & 0 & 0.594996259 & 0.0055482 \\
\hline
\end{tabular}

Tabela 5.2 Resultados do Estimador Ótimo $\left(\hat{\theta}_{*}\right)$ Balanceado Ponderado, fazendo variar o Parâmetro de Forma $a$.

\begin{tabular}{|c|l|l|l|l|}
\hline & $a=0.01$ & $a=0.05$ & $a=0.07$ & $a=0.09$ \\
\hline$w=0.00$ & 5.567613 & 5.562053 & 5.558777 & 5.555513 \\
\hline$w=0.25$ & 7.696599 & 7.682223 & 7.668010 & 7.649404 \\
\hline$w=0.50$ & 7.696998 & 7.692163 & 7.687304 & 7.680823 \\
\hline$w=0.75$ & 7.697131 & 7.695515 & 7.693881 & 7.691688 \\
\hline$w=1.00$ & 7.697198 & 7.697198 & 7.697198 & 7.697198 \\
\hline
\end{tabular}

\subsection{Conclusão}

Da Tabela 5.1, podemos observar algumas vantagens utilizando a Função de Perda Balanceada Ponderada (FPBP). Por exemplo, para $w=0.25$ e utilizando a expressão (5.15) o resultado obtido é 0.45134874 , concluindo que o risco é aumentado em $45.134874 \%$

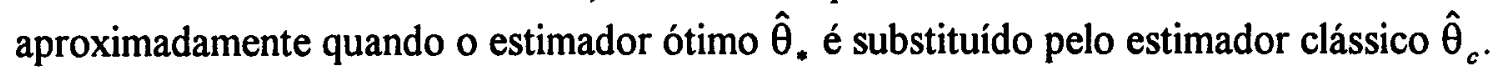


Na segunda coluna, da tabela 5.1, temos os resultados do estimador ótimo que reflete simultaneamente o ajuste do modelo e a precisão do estimador. Também quando fazemos $w=1$, o estimador ótimo é igual ao estimador clássico, $\hat{\theta}_{*}=\hat{\theta}_{c}$, e sua perda relativa é igual a zero.

Quando o estimador ótimo é substituído pelo estimador Bayesiano as conclusões com respeito a perda relativa são análogas as anteriores.

$\mathrm{Na}$ última coluna da Tabela 5.1, são apresentadas as estimativas da função de confiabilidade baseado no ajuste do modelo e na função de perda Linex.

Verificamos numericamente, que o parâmetro de forma $a$ não influi substancialmente na obtenção do estimador ótimo balanceado. A análise numérica sugere que o estimador é robusto em relação a parâmetro de forma da função de perda Linex. (ver Tabela 5.2) 


\section{CAPÍTULO 6}

\section{Conclusões e Perspectivas Futuras}

seguir:

No decorrer deste trabalho obtivemos algumas conclusões que serão resumidas a

Explorando o uso dos métodos Bayesianos, conseguimos obter resultados mais realísticos nas inferências pontuais quando se utiliza funções de perdas assimétricas. Porém, estas funções de perdas são mais complexas que às perdas simétricas, por esta razão foi preciso utilizar algoritmos na obtenção dos resultados. Por outro lado, também obtivemos estimativas pontuais usando função de perda quadrática, tais resultados foram úteis para ser comparados em termos de risco e risco de Bayes com o resultado da função de perda assimétrica dado no capítulo 4 .

Estimativas para a confiabilidade foram encontradas usando diferentes densidades a priori. Alguns resultados obtidos no capítulo 2, coincidem com os resultados clássicos, pois as densidades a priori consideradas foram não-informativas.

É importante salientar os resultados obtidos no capítulo 5, quando se faz uso da Função de Perda Balanceada Ponderada (FPBP), pois esta perda estabelece um compromisso entre a estatística clássica e a Bayesiana.

Como continuação desta tese, pretendemos futuramente pesquisar outros tópicos, tais como:

1. Estender os resultados obtidos do capítulo 5, utilizando a Função de Perda Balanceada Ponderada com os dados censurados gerados pelo modelo da distribuição de Weibull.

2. Aplicação da Função de Perda Balanceada Ponderada (FPBP) e Linex no problema de seleção, introduzido por Marsh, L.; Zellner A. (1994). 


\section{Apêndice A}

\section{Algumas Integrais Usuais}

1. $\int_{0}^{\infty} \frac{T^{(n-\beta)-1} \theta^{(n-\beta)} e^{-\theta T}}{\Gamma(n-\beta)} d T=1$

2. $\int_{0}^{\infty} \frac{T^{(n-2 \beta)-1} \theta^{(n-2 \beta)} e^{-\theta T}}{\Gamma(n-2 \beta)} d T=1$

3. $\int_{0}^{\infty} \frac{(b+s)^{(a+n)} \theta^{(a+n-1)} e^{-(b+s) \theta}}{\Gamma(a+n)} d \theta=1$

4. $\int_{0}^{\infty} \frac{\theta^{(\alpha+\beta+a+n)}(b+s)^{(\alpha+\beta+a+n)-1} e^{-(b+s) \theta}}{\Gamma(\alpha+\beta+a+n)} d \theta=1$

5. $\int_{0}^{\infty} \frac{\theta^{(\alpha+a+n)}(b+s)^{(\alpha+\beta+n)-1} e^{-(b+s) \theta}}{\Gamma(\alpha+a+n)} d \theta=1$ 


\section{Apêndice B}

\section{Método Newton-Raphson}

Suponhamos que desejamos encontrar a raiz $\hat{\theta}$ da equação $g(\theta)=0$. Seja $\theta_{0}$ um valor fixo para $\theta$ e expandindo $g(\theta)$ através de uma série de Taylor no ponto $\theta_{0}$, temos:

$$
g(\theta)=g\left(\theta_{0}\right)+\left(\theta-\theta_{0}\right) g^{\prime}\left(\theta_{0}\right)+\frac{\left(\theta-\theta_{0}\right)^{2} g^{\prime \prime}\left(\theta_{0}\right)}{2 !}+\ldots
$$

Para $\left|\theta-\theta_{0}\right|$ pequeno, os termos n-ésimos na expressão acima são pequenos e eliminando estes termos, temos:

$$
g(\theta) \approx g\left(\theta_{0}\right)+\left(\theta-\theta_{0}\right) g^{\prime}\left(\theta_{0}\right)
$$

Portanto, aproximamos $g(\theta)$ por uma função linear de $\theta$. Como $g(\theta)=0$, obtemos:

$$
g\left(\theta_{0}\right)+\left(\theta-\theta_{0}\right) g^{\prime}\left(\theta_{0}\right) \approx 0
$$

ou,

$$
\theta_{1} \approx \theta_{0}-\frac{g\left(\theta_{0}\right)}{g^{\prime}\left(\theta_{0}\right)}
$$

No método Newton-Raphson, tomamos $\theta_{0} \operatorname{como}$ sendo uma condição inicial para $\theta$ e o valor de $\theta$ na etapa seguinte, $\theta_{1}$, é dado pela expressão acima.

Repetindo os cálculos, tomando $\theta_{1}$ como condição inicial temos:

$$
\theta_{2}=\theta_{1}-\frac{g\left(\theta_{1}\right)}{g^{\prime}\left(\theta_{1}\right)}
$$


Continuamos este procedimento até $\theta_{i+1} \approx \theta_{i}$, neste caso, $g\left(\theta_{i}\right)=0$ e a raíz $\hat{\theta}$ é dada por $\theta_{i}$. 


\section{Apêndice C}

\section{Programa para encontrar o estimador ótimo Balanceado Ponderado, para diferentes valores de $w$}

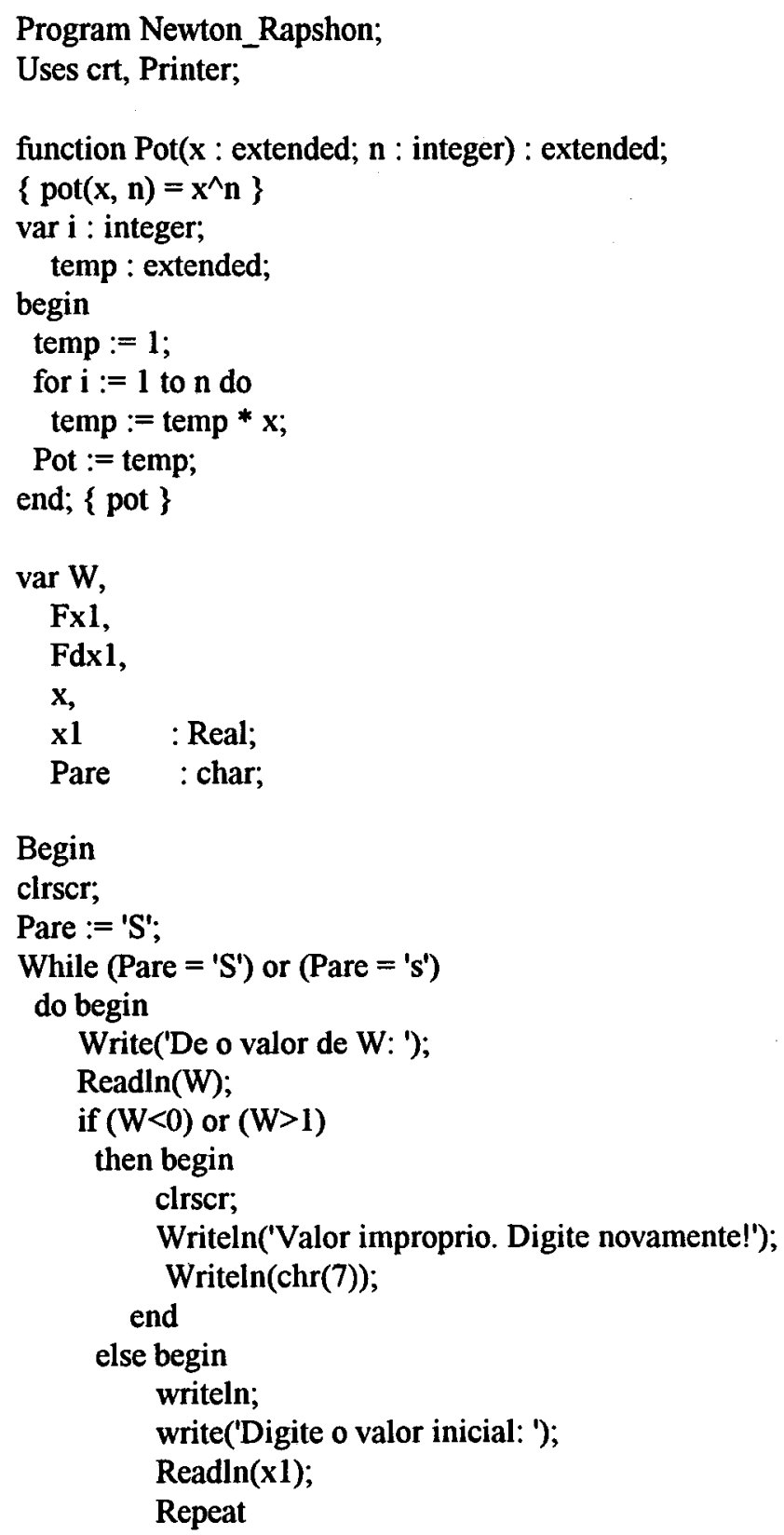




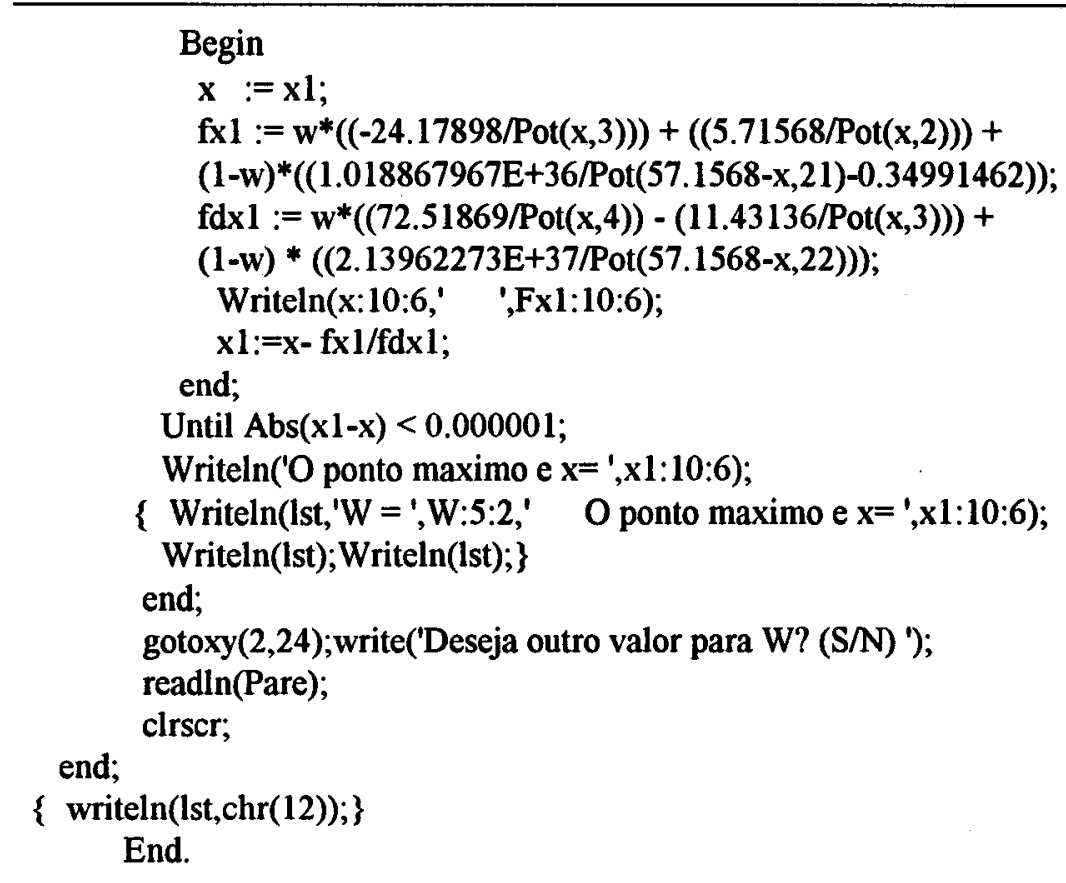

\title{
Programa Gerando Dados para uma Exponencial e obtendo o Estimador Ótimo Balanceado Ponderado (Software Minitab)
}

\author{
let $\mathbf{k l}=\mathbf{1 1}$ \\ let $\mathrm{k} 10=1$ \\ let $\mathrm{k} 11=1$ \\ let $\mathbf{k 9}=1$ \\ let $k 7=1$ \\ let $\mathrm{k} 15=1$ \\ let $\mathrm{k} 16=5$ \\ let $\mathrm{k} 17=0.25$ \\ let $\mathrm{k} 18=0.50$ \\ let $\mathrm{k} 19=0.75$ \\ Store 'Simul' \\ randon $15 \mathrm{cl}$; \\ expo 3. \\ sort cl c2 \\ delete 1: $3 \mathrm{c} 2$ \\ end \\ Store 'Amostra' \\ let $\mathrm{c} 2(\mathrm{kl})=\mathrm{c} 2(10)$ \\ let $\mathbf{k} \mathbf{l}=\mathbf{k} \mathbf{l}+\mathbf{1}$ \\ end \\ Store 'Soma' \\ print $\mathrm{c} 2$ \\ sum c2 k2
}


let $\mathrm{k} 3=\mathrm{k} 2 / 10$

let $\mathrm{c} 4=(\mathrm{c} 2-\mathrm{k} 3)^{* * 2}$

sum $\mathrm{c} 4 \mathrm{k} 4$

let $\mathrm{k} 5=\mathrm{k} 4 / 10$

let $\mathrm{k} 6=\left(\mathrm{k} 5+1.5^{*}(\mathrm{k} 3 * * 2)\right)$

Store ' $N R 1$ '

let $\mathrm{k} 20=\mathrm{k} 15^{*}\left(-2 * \mathrm{k} 6 / \mathrm{k} 16^{* *} 3+3 * \mathrm{k} 3 / \mathrm{k} 16^{* *} 2\right)+(1-\mathrm{k} 15) *(\operatorname{expo}(-1) *(\mathrm{k} 2 * * 10) * 10 /((\mathrm{k} 2-\mathrm{k} 16) * * 11)-10 / \mathrm{k} 2)$

let $\left.\mathrm{k} 21=\mathrm{k} 15^{*}\left(6 * \mathrm{k} 6 / \mathrm{k} 16^{* * 4}-6^{*} \mathrm{k} 3 / \mathrm{k} 16^{* * 3}\right)+(1-\mathrm{k} 15)^{*}\left(\operatorname{expo}(-1)^{*}(\mathrm{k} 2 * * 10)^{*} 10^{* 11} /(\mathrm{k} 2-\mathrm{k} 16)^{* * 12}\right)\right)$

let $\mathrm{k} 22=\mathrm{k} 16-\mathrm{k} 20 / \mathrm{k} 21$

let $\mathrm{k} 16=\mathrm{k} 22$

let $\mathrm{cl}(\mathrm{k} 10)=\mathrm{k} 16$

let $\mathrm{cll}(\mathrm{k} 10)=\mathrm{k} 20$

end

Store 'NR2'

let $\mathrm{k} 30=1-\mathrm{k} 15$

let $\mathrm{k} 20=\mathrm{k} 30^{*}\left(-2 * \mathrm{k} 6 / \mathrm{k} 16 * * 3+3 * \mathrm{k} 3 / \mathrm{k} 16^{* * 2}\right)+(1-\mathrm{k} 30)^{*}\left(\operatorname{expo}(-1)^{*}(\mathrm{k} 2 * * 10) * 10 /((\mathrm{k} 2-\mathrm{k} 16) * * 11)-10 / \mathrm{k} 2\right)$

let $\mathrm{k} 21=\mathrm{k} 30^{*} \mathrm{k} 15^{*}\left(6^{*} \mathrm{k} 6 / \mathrm{k} 16^{* *} 4-6^{*} \mathrm{k} 3 / \mathrm{k} 16^{* * 3}\right)+(1-\mathrm{k} 30)^{*}\left(\operatorname{expo}(-1)^{*}(\mathrm{k} 2 * * 10)^{*} 10^{*} 11 /\left((\mathrm{k} 2-\mathrm{k} 16)^{* *} 12\right)\right)$

let $\mathrm{k} 22=\mathrm{k} 16-\mathrm{k} 20 / \mathrm{k} 21$

let $\mathrm{k} 16=\mathrm{k} 22$

let $\operatorname{cls}(\mathbf{k 1 0})=\mathbf{k 1 6}$

let $\mathrm{c} 16(\mathrm{k} 10)=\mathrm{k} 20$

end

Store 'NR3'

let $\mathrm{K} 20=\mathrm{k} 17 *\left(-2 * \mathrm{~K} 6 / \mathrm{k} 16^{* * 3}+3 * \mathrm{k} 3 / \mathrm{k} 16^{* *} 2\right)+(1-\mathrm{k} 17)^{*}\left(\exp (-1)^{*}(\mathrm{k} 2 * * 10)^{*} 10 /\left((\mathrm{k} 2-\mathrm{k} 16)^{* *} 11\right)-10 / \mathrm{k} 2\right)$

let $\mathrm{k} 21=\mathrm{k} 17^{*}\left(6^{*} \mathrm{k} 6 / \mathrm{k} 16^{* *} 4-6^{*} \mathrm{k} 3 / \mathrm{k} 16^{* *} 3\right)+(1-\mathrm{k} 17)^{*}\left(\exp (-1)^{*}(\mathrm{k} 2 * * 10)^{*} 10^{*} 11 /\left((\mathrm{k} 2-\mathrm{k} 16)^{* *} 12\right)\right)$

let $\mathrm{k} 22=\mathrm{k} 16-\mathrm{k} 20 / \mathrm{k} 21$

let $\mathrm{k} 16=\mathrm{k} 22$

let $\mathrm{c} 20(\mathrm{~K} 11)=\mathrm{k} 16$

let $\mathrm{c} 21(\mathrm{k} 11)=\mathrm{k} 20$

end

Store 'NR4'

let $\mathrm{k} 20=\mathrm{k} 18 *\left(-2 * \mathrm{k} 6 / \mathrm{k} 16^{* *} 3+3 * \mathrm{k} 3 / \mathrm{k} 16^{* *} 2\right)+(1-\mathrm{k} 18)^{*}\left(\exp (-1) *(\mathrm{k} 2 * * 10)^{*} 10 /(\mathrm{k} 2-\mathrm{k} 16)^{* *} 11\right)-$ $10 / \mathrm{k} 2$ )

let $\mathrm{k} 2 \mathrm{l}=\mathrm{k18}\left(6^{*} \mathrm{k} 6 / \mathrm{k} 16^{* *} 4-6^{*} \mathrm{k} 3 / \mathrm{k} 16^{* *} 3\right)+(1-\mathrm{k} 18)^{*}\left(\exp (-1)^{*}(\mathrm{k} 2 * * 10)^{*} 10^{*} 11 /\left((\mathrm{k} 2-\mathrm{k} 16)^{* *} 12\right)\right)$

let $\mathrm{k} 22=\mathrm{k} 16-\mathrm{k} 20 / \mathrm{k} 21$

let $\mathbf{k} 16=\mathbf{k} 22$

let $\mathrm{c} 22(\mathrm{k} 9)=\mathrm{k} 16$

let $\mathrm{c} 23(\mathrm{k} 9)=\mathrm{k} 20$

end

Store 'NR5'

let $\mathrm{k} 20=\mathrm{k} 19 *\left(-2 * \mathrm{~K} 6 / \mathrm{k} 16^{* * 3}+3 * \mathrm{k} 3 / \mathrm{k} 16^{* *} 2\right)+(1-\mathrm{k} 19) *\left(\exp (-1) *(\mathrm{~K} 2 * * 10) * 10 /\left((\mathrm{k} 2-\mathrm{k} 16)^{* * 11}\right)-10 / \mathrm{k} 2\right)$

let $\mathrm{k} 21=\mathrm{k} 19 *\left(6 * \mathrm{k} 6 / \mathrm{k} 16^{* *} 4-6 * \mathrm{k} 3 / \mathrm{k} 16^{* *} 3\right)+(1-\mathrm{k} 19) *\left(\exp (-1) *(\mathrm{k} 2 * * 10)^{* 10} 10^{* 11}\left((\mathrm{k} 2-\mathrm{k} 16)^{* *} 12\right)\right)$

let $k 22=\mathrm{k} 16-\mathrm{k} 20 / \mathrm{k} 21$

let $\mathrm{k} 16=\mathrm{k} 22$

let $\mathrm{c} 24(\mathrm{k} 7)=\mathrm{k} 16$

let $\mathrm{c} 25(\mathrm{~K} 7)=\mathrm{k} 20$

end 
Store 'Tudo'

exec 'Simul'

exec 'Amostra' 5

exec 'Soma'

let $\mathrm{c5}(\mathrm{k} 10)=\mathrm{k} 3$

let $\mathrm{c} 6(\mathrm{k} 10)=\mathrm{k} 5$

let $\mathrm{k} 1=11$

exec 'NR1' 15

let $k 16=3$

exec 'NR2' 10

let $\mathrm{k} 10=\mathrm{k} 10+1$

exec 'NR3' 15

let $\mathbf{k} 16=3$

let $k 11=k 11+1$

exec 'NR4' 15

let $\mathrm{k} 16=3$

let $\mathrm{k} 9=\mathrm{k} 9+1$

exec 'NR5' 15

let $\mathbf{k} 16=3$

let $\mathbf{k} 7=\mathbf{k} 7+1$

end 


\section{Referências Bibliograficas}

BARNARD, G.A. (1954). Sampling Inspeccion and Statistical Decisions. Journal Royal Statistical Society. 16, 151-174.

BASU, A.; EBRAHIM, N. ( 1988). Bayesian Approach to Life and Reliability Estimation Using Asymmetric Loss Funtion. Air Force Office of Scientific Research (AFOSR). Technical Report No 144.

BHATTACHARYA, S.K. (1967). Bayesian Approach to life Testing and Reliability Estimation. American Statistical Association Journal. 3, pp. 50-62

BERGER, J. (1985). Stastistical Decision Theory and Bayesian Analysis. (2nd. Ed). Springer Series in Statistics. Springer Verlag.

BOLFARINE, H.; RODRIGUES, J.; ACHCAR, J.A. (1991). Análise de Sobrivência. Rio de Janeiro, IME - UFRJ, 112 pag's. (Notas do Minicurso da II Escola de Modelos de Regresão).

BOX, G.E.P.; TIAO, G.C. (1973). Bayesian Inference Statistical Analysis. New York: Addison - Wesley.

BROW, P. (1984). Notes in Decision Theory. College, London.

CANFIELD, R.V. (1970). A Bayesian Approach to Reliability Estimation Using a Loss Function. IEEE Transactions on Reliability, Vol. R-19, pp.13-16.

CHIOU, P. (1993). Empirical Bayes Shrinkage Estimation of Reliability in the exponential Distribution. Commun Statist. - Theory Math, 22(5), 1483-1494.

DE GROOT, M.H. (1970). Optimal Statistical Decisions. MC Graw-Hill, New York.

EL-SAYYAD, G.M. (1967). Estimation of the Parameter of an Exponential Distribution. Journal of the Royal Statistical Society, Serie B, Vol. 29, pp.57-61. 
FERGUSON, T.S. (1967). Mathematical Statistics, A. Decision Theoretic Approach. Springer-Verlarg.

GIRSHICK, M.A.; SAVAGE, L. J. (1951). Bayes an Minimax Estimates for Quadratic Loss Funtions. Proc. 2nd Berkeley Symp. Math. Statist. \& Prob., pp. 53-67.

GOODMAN, L.A. (1953). A Simple Methods for Improving Some Estimators. Ann. Math. Statist, 24,114-117.

JAMES, W.; STEIN, C. (1961). Estimation with quadratic loss. Proc. Fourth Berkeley Symp. Math. Statist Prob, 1, pp. 331-319.

KALBFLEISCH, J. D. (1980). Probability e Statistical Inference. Volume 2: Statistical Inference. Springer-Verlag.

LAWLESS, J. F. (1982). Statistical Models and Methods for Lifetime Data. New York, John Wiley \& Sons.

LEHMANN, E.L. (1951). Theory of Point Estimation. Wiley, New York.

LINDLEY, D.V. (1971). Making Decisions. Wiley-Intercience.

MARTZ, H. F.; WALLER, R. A. (1982). Bayesian Reliability Analysis. New York, John Wiley \& Sons

PANDEY, B.N.; OMKAR, R. (1992). Bayesian Estimation of Mean and Square of Mean of Normal Distribution Using Linex Loss Funtion. Comun Statist.- Theory Math, 21(12), 3369-3391.

PERICCHI, L. R.(1986). Notes in Decision Theory. Manuscrito de curso en el Imperial college, London.

PERICCHI, L. R. (1992). Teoria Estadistica de Decisión e Análisis Bayesiano. Centro de Estadística e Software Matemático (CESMa).

PRESS, S. J. (1989). Bayesian Statistics Models and Applications. New York, JohnWiley \& Sons.

PUGH, E.L. (1963). The Best Estimate of Reliability in the Exponencial Case. Operation Res, Vol . 11, pp. 57-61. 
RAIFFA, H.; SCHLAIFER, R. (1961). Applied Statistical Decisions Theory. Boston: Graduate School of Business Administration, Havard University.

ROBBINS, H. (1963). The Emperical Bayes Approach to Statistical Decision Problems. Ann. Math. Statis. 29. pp. 1-19.

RODRIGUES, J. ZELLNER, A. (1991). Weighted Balancead Loss Funtion for the exponential Mean time to Failure. Artigo publicado em communications in statistics.

RODRIGUES, J. (1993). Bayesian Estimation for a Normal Mean Parameter Using Linex Loss Funtion. Artigo aceito na revista Test.

SACHABE, H. (1991). Bayes Estimates under Asymmetrics Loss. IEEE Transactions on Reliability, Vol.40, pp. 63-67.

SAVAGE, L. J. (1954). The foundations of Statics. Wiley, New York.

SINHA, S.K.; KALE, B.K.(1980). Life Testing and Reliability Estimation. Wiley Eastern limited, New Delhi.

VARIAN, H.R. (1975). A Bayesian Approach to Ral Estate Assessment, in Studies in Bayesians Econometrics and Statistics in Honor of Leonard J. Savage, eds. Stephen E. Fienberg and Arnold Zellner. Amsterdam: Nort-Holland, pp. 195-208.

WALD, A. (1950). Statistical Decisions Funtions. Wiley, New York.

WALLEY, P. (1897). Notes in Decisions Theory. Univesidade de Cornell, U.S.A.

WETHERILL, G.B. (1961). Bayesian Sequential Analysis. Biometrica, 48, 281- 282.

ZELLNER, A.; GEISEL. M.S. (1968). Sensitivity of Control to Uncertainty adm Form of the criterion Funtion. In the Future of statistics, ed. Donal G. Watts, New York: Academic press, pp. 269-289.

ZELLNER, A. (1987). Bayesian Estimation and Prediction Using Asymmetric Loss Functions. Journal of the American Statistical Association, Vol. 81, 446-451.

ZELLNER, A. (1988). Bayesian Analysis en Econometrics. Journal of Econometrics 37, pags. 27-50. 
ZELLNER, A. (1991). Bayesian and Non-Bayesain Estimation Using Balanced Loss Funtions. H.G.B. Alexandre Research Foudation Graduate School of Business University of Chicago. 